\title{
Noise Performance and Long-Term Stability of Near- and Mid-IR Gas-Filled Fiber
} Raman Lasers

Wang, Yazhou; Adamu, Abubakar; Dasa, Manoj Kumar; Antonio-Lopez, Jose Enrique; Habib, Md Selim; Correa, Rodrigo Amezcua; Bang, Ole; Markos, Christos

Published in:

Journal of Lightwave Technology

Link to article, DOI:

10.1109/JLT.2021.3067514

Publication date:

2021

Document Version

Peer reviewed version

Link back to DTU Orbit

Citation (APA):

Wang, Y., Adamu, A., Dasa, M. K., Antonio-Lopez, J. E., Habib, M. S., Correa, R. A., Bang, O., \& Markos, C. (2021). Noise Performance and Long-Term Stability of Near- and Mid-IR Gas-Filled Fiber Raman Lasers. Journal of Lightwave Technology, 39(11), 3560-3567. https://doi.org/10.1109/JLT.2021.3067514

\section{General rights}

Copyright and moral rights for the publications made accessible in the public portal are retained by the authors and/or other copyright owners and it is a condition of accessing publications that users recognise and abide by the legal requirements associated with these rights.

- Users may download and print one copy of any publication from the public portal for the purpose of private study or research.

- You may not further distribute the material or use it for any profit-making activity or commercial gain

- You may freely distribute the URL identifying the publication in the public portal 


\title{
Noise Performance and Long-Term Stability of Near- and Mid-IR Gas-Filled Fiber Raman Lasers
}

\author{
Yazhou Wang (i), Abubakar Isa Adamu (i), Manoj K. Dasa, Jose E. Antonio-Lopez, Md. Selim Habib ${ }^{(\mathbb{B},}$ \\ Rodrigo Amezcua-Correa, Ole Bang, and Christos Markos (i)
}

\begin{abstract}
Stimulated Raman scattering (SRS) enabled by the emerging gas-filled low-loss anti-resonant hollow-core fiber (ARHCF) technology opens up a competitive way towards the development of novel lasers in the molecular fingerprint region. In this article, the characteristics of noise and long-term stability of near- and mid-infrared (near-IR and mid-IR) gas-filled fiber Raman lasers have been investigated for the first time. The results reveal that an increase in Raman pulse energy is associated with a decrease in noise, and that the relative pulse peak intensity noise (RIN) is always lower than the relative pulse energy noise (REN). We also demonstrate that long-term drift of the pulse energy and peak power are directly linked with the high amount of heat release during the Raman Stokes generation. The demonstrated noise and long-term stability performance provide necessary references for potential spectroscopic applications as well as further improvements of the emerging IR gas-filled ARHCF Raman laser technology.
\end{abstract}

Index Terms-Laser noise, laser stability, optical fiber lasers, Raman lasers.

\section{$\mathbf{T}$}

\section{INTRODUCTION}

TE emerging gas-filled hollow-core fiber (HCF) Raman laser technology has attracted enormous attention due to

Manuscript received January 4, 2021; revised March 3, 2021; accepted March 11,2021 . This work was supported in part by Danmarks Frie Forskningsfond Hi-SPEC under the Grant 8022-00091B, Lundbeck Foundation under MultiBRAIN project (R276-2018-869), VILLUM FONDEN under Grant 36063, Innovation Fund Denmark UVSUPER under Grant 8090-00060A, ECOMETA under the Grant 6150-00030B, and in part by U.S. ARO under Grant W911NF19-1-0426. (Corresponding authors: Yazhou Wang; Christos Markos.)

Yazhou Wang, Abubakar Isa Adamu, and Manoj K. Dasa are with the Department of Photonics Engineering, Technical University of Denmark, DK- 2800 Lyngby, Denmark (e-mail: yazwang@fotonik.dtu.dk; abisa@fotonik.dtu.dk; manda@fotonik.dtu.dk).

Ole Bang is with the Department of Photonics Engineering, Technical University of Denmark, DK- 2800 Lyngby, Denmark, and with NKT Photonics, 3460 Birkerød, Denmark, and with the NORBLIS IVS, 2830 Virum, Denmark (e-mail: oban@fotonik.dtu.dk).

Christos Markos is with the Department of Photonics Engineering, Technical University of Denmark, DK- 2800 Lyngby, Denmark, and also with the NORBLIS IVS, 2830 Virum, Denmark (e-mail: chmar@ fotonik.dtu.dk).

Jose E. Antonio-Lopez and Rodrigo Amezcua-Correa are with the College of Optics and Photonics, University of Central Florida, Orlando FL-32816 USA (e-mail: jealopez@creol.ucf.edu; r.amezcua@creol.ucf.edu).

Md. Selim Habib is with the Department of Electrical and Computer Engineering, Florida Polytechnic University, Lakeland FL-33805 USA (e-mail: mhabib@floridapoly.edu).

Color versions of one or more figures in this article are available at https: //doi.org/10.1109/JLT.2021.3067514.

Digital Object Identifier 10.1109/JLT.2021.3067514 its compelling features that enable efficient stimulated Raman scattering (SRS) process, high damage threshold, broad transmission range, compact fiber structure, high freedom on wavelength selection, etc. [1]-[3]. Especially, with the recent advent of low loss anti-resonant HCF (ARHCF) technology [4]-[6], the laser wavelength has been extended to the mid-infrared (mid-IR) region where the high silica loss could be significantly mitigated by confining most power of the laser beam within the fiber core (gas) region [7]-[9]. A series of gas-filled fiber Raman lasers have been reported from the UV to mid-IR region [7], [10]-[15]. Despite their unique advantages compared to other counterparts, high noise is an intrinsic issue of Raman lasers since the SRS process is usually initiated by the quantum noise [16]-[18]. The noise of Raman laser has extensively been investigated in the frame of a bulk gas cell usually filled with hydrogen $\left(\mathrm{H}_{2}\right)$ [19]-[22], but yet remains unexplored for the gas-filled fiber technology. The noise characteristics of the latter is worth further investigation since the SRS process in gas-filled HCF is much more efficient than the conventional bulk gas cell structure. Besides, for the Raman Stokes emission involving a large photon energy difference between the pump and Stokes, the energy of the released phonon is high and thus could easily lead to a large amount of heat release within the core region of the HCF, where the gas circulation speed is much slower than its counterpart of using bulk gas cell. As a result, the temperature and therefore the Raman gain coefficient will be directly affected. In the last few years, several efficient Raman lasers operating at $\sim 4 \mu \mathrm{m}$ wavelength have been reported with $\mathrm{H}_{2}$-filled ARHCFs by using $1.5 \mu \mathrm{m}$ fiber laser as a pump [7], [10], [11], where the generation of one Stokes photon is accompanied by phonon emission with up to $\sim 60 \%$ of pump photon energy. Consequently, the heat pulse energy of these mid-IR Raman lasers is calculated to be $\mu \mathrm{J}$ level, which could impose a significant impact on the laser stability. However, as an emerging laser technology, these issues have not been investigated yet.

In this work, we thoroughly investigated the noise and stability performance of two different Raman lasers based on an $\mathrm{H}_{2}$-filled ARHCF, at $1.68 \mu \mathrm{m}$ and $4.2 \mu \mathrm{m}$. The Raman lasers were generated by pumping with a custom-made linearly polarized fiber laser at $1532.8 \mathrm{~nm}$. The pump laser has a pulse energy of $\sim 80 \mu \mathrm{J}$, pulse duration of $7 \mathrm{~ns}$, repetition rate of $8 \mathrm{kHz}$, and linewidth of $\sim 0.06 \mathrm{~nm}$. The ARHCF is designed with two transmission windows around $1.3-1.8 \mu \mathrm{m}$, and $2.5-4.5 \mu \mathrm{m}$, separated by a resonance peak at $\sim 2 \mu \mathrm{m}[11]$. 


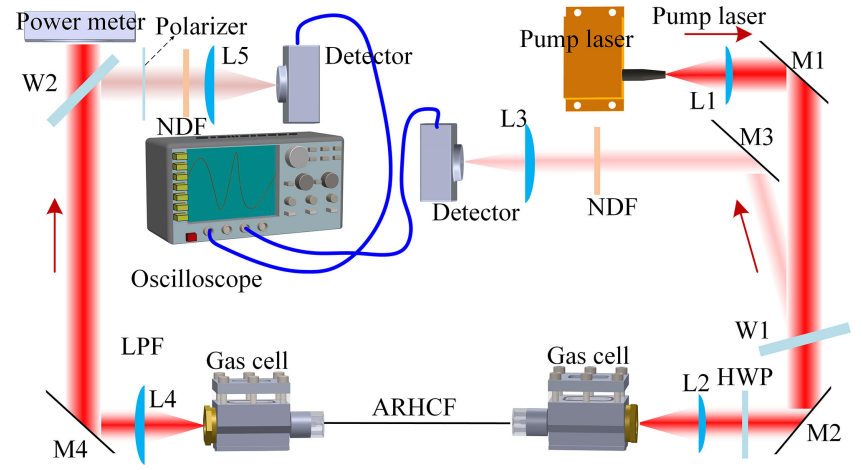

Fig. 1. Noise measurement setup of gas-filled fiber Raman laser. M1-M4 are silver mirrors. L1-L3 are C-coated plano-convex silica lenses, L4-L5 are uncoated plano-convex $\mathrm{CaF}_{2}$ lenses. $\mathrm{W} 1$ and $\mathrm{W} 2$ are respectively optical wedge and window used to extract a small part of the laser beam for pulse detection with photodetectors.

\section{EXPERIMENT SETUP AND METHOD}

Fig. 1 shows the details of the experimental setup used for this investigation. $\mathrm{H}_{2}$ is filled into the ARHCF through two gas cells. At the pressure range of 5-20 bar, $\mathrm{H}_{2}$ inside the ARHCF quickly reaches to equilibrium state within one minute. The fiber length was optimized to $3.95 \mathrm{~m}$, to obtain a maximum

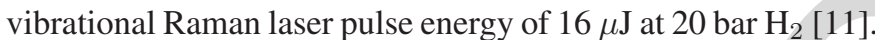
The output from the $\mathrm{H}_{2}$-filled ARHCF is first collimated and then the residual pump is removed by a long-pass filter (LPF). The remaining $4.22 \mu \mathrm{m}$ Raman part is passed through a $\mathrm{CaF}_{2}$ window ( $\sim 95 \%$ transmission at $4.22 \mu \mathrm{m}$ ) and monitored by a thermal power meter. A small part of the Raman laser reflected from the front surface of the $\mathrm{CaF}_{2}$ window is focused onto a photodetector (PDAVJ10, Thorlabs) which is connected to an oscilloscope (Teledyne Lecroy HD09000) for pulse profile monitoring. The pulse duration of the Raman laser is estimated to be a few nanoseconds, which is comparable with the rise time of the photodetector $(\sim 3.5 \mathrm{~ns})$, as a result, the Raman laser pulse profile couldn't be accurately measured. Here we use the deconvolution method to retrieve the original pulse profile, as described in Appendix A. Based on this method, we found that both pulse energy and peak power of the Raman laser could be correctly and respectively reflected by the pulse area and peak intensity measured from the oscilloscope, as long as the Raman power is attenuated to the linear region of the photodetector. Because the $4.22 \mu \mathrm{m}$ Raman line here has a high polarization extinction ratio (PER) $(>20 \mathrm{~dB}$ ) (see Appendix B), a polarizer was used to continuously attenuate the average power, so that most of the pulses could be appropriately measured in the linear response region of the photodetector. The characterization of the photodetector is provided in Appendix A. The pump laser was also simultaneously monitored by focusing a minor part of the pump into a near-infrared (near-IR) photodetector (DET01CFC, Thorlabs).

From the setup in Fig. 1, due to the fact that the pump is linearly polarized, the rotational Raman line at $1.68 \mu \mathrm{m}$ has a near-zero power (see the measured spectrum in Fig. 2) and thus negligible influence on the generation of a vibrational Raman line at $4.22 \mu \mathrm{m}$. Nevertheless, for the purpose of comparison,

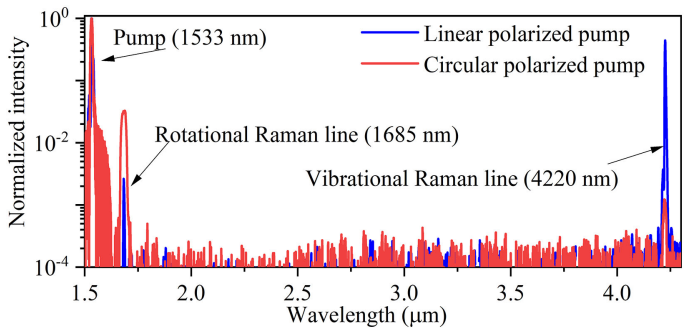

Fig. 2. Spectra comparison of the Raman lines with linear and circular polarized pumps.

we also monitored the noise and long-term stability of the rotational Raman line generated at $1.68 \mu \mathrm{m}$ when replacing the half-wave plate (HWP) with a quarter-wave plate (QWP) in front of the ARHCF to make the pump light circularly polarized [23]. When using circularly polarized pump light, the $\mathrm{H}_{2}$ pressure was reduced to 8 bar to suppress the vibrational Raman line, which finally provided a quantum efficiency and pulse energy of the rotational Raman line of $45 \%$ and $27 \mu \mathrm{J}$, respectively, whereas the average power of the vibrational Raman line was measured to be less than $1 \mathrm{~mW}$ (see Fig. 2). Then, the pulse profile of the rotational Raman line was precisely measured by the other near-IR photodetector with the same parameters as the one used for monitoring the pump. Because of the circular polarization of the rotational Raman line, the polarizer was replaced by a continuously variable near-IR neutral density filter (NDF) (NDL-10C-2, Thorlabs), to precisely attenuate the pulse peak power.

\section{RESULTS AND ANALYSIS}

\section{A. Noise Performance}

In the noise measurement, we collected a train of 10000 Raman pulses and corresponding pump pulses, for each pump power level. The influence of the pump noise is suppressed by discarding the pump pulses whose peak intensity falls outside $2 \%$ of its average value. After the data processing, the number of pairs is reduced to $\sim 9500$, and the relative noise (defined as the ratio of the standard deviation to the mean value) of the pump peak intensity is reduced from $\sim 1.7 \%$ to $\sim 1 \%$. Fig. 3(a) and 3(b) present the measured relative pulse energy noise (REN) and relative pulse peak intensity noise (RIN) of the vibrational and rotational Raman lasers. It shows that both of them gradually decrease, and in Fig. 3(a) they finally approach a stable level as the Raman laser power exceeds $\sim 80 \mathrm{~mW}$ (corresponding to $\sim 45 \%$ quantum efficiency). This is attributed to the stabilization effect of Stokes pulse energy induced by the depletion of pump power as well as $\mathrm{H}_{2}$ molecules in the ground state during the SRS [24]. Meanwhile, at the same pulse energy level, it can be seen that the RIN is always lower than the REN. For instance, at the highest power of $132.8 \mathrm{~mW}$ in Fig. 3(a), the REN is $16.8 \%$, whereas the RIN is as low as $2.9 \%$. This difference is supposed to be caused by the dependence of the SRS efficiency on the light intensity: the SRS process in the peak region of the Raman pulse is more efficient than at its edge regions, whereas high SRS 

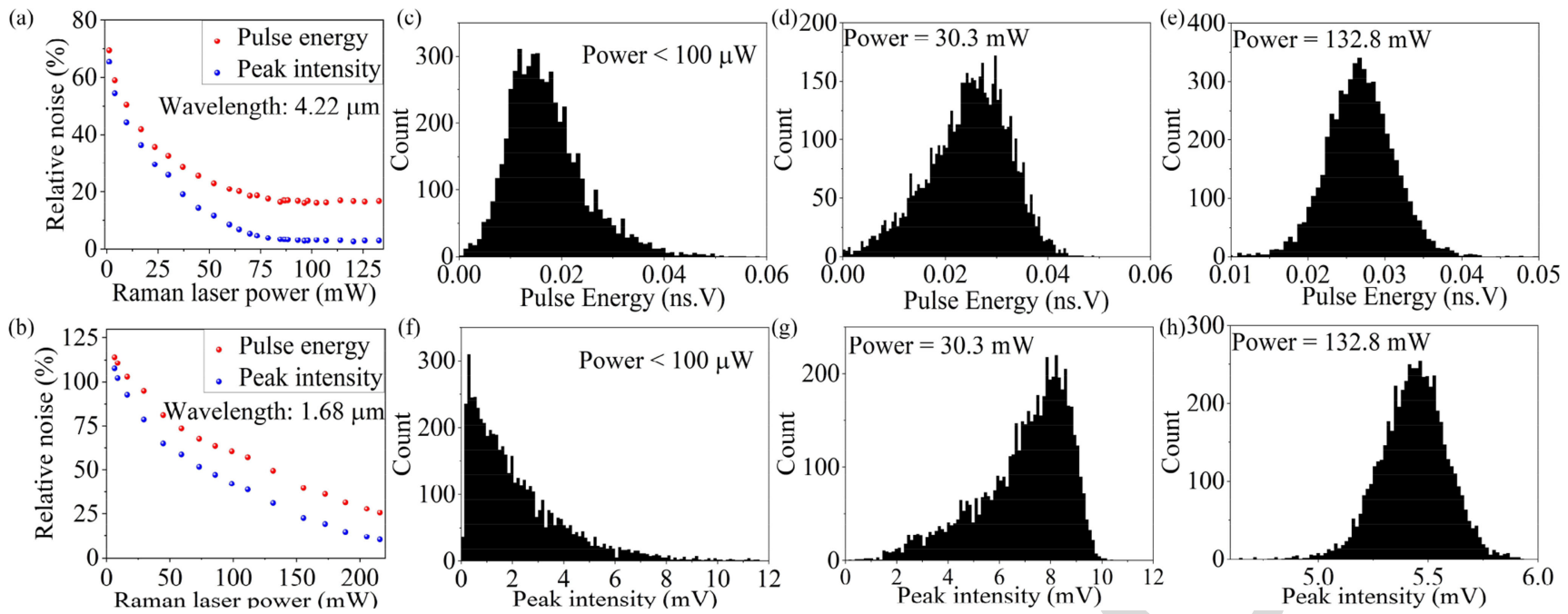

Fig. 3. (a) and (b) are the measured REN and RIN of the $4.22 \mu \mathrm{m}$ and $1.68 \mu \mathrm{m}$ Raman lines as a function of their average powers, respectively. (c)-(e) are histograms of measured pulse energy at different levels of output Raman laser power (4.22 $\mu \mathrm{m})$. (f)-(h) are histograms of measured pulse peak intensity corresponding to (c)-(e).

efficiency is associated with the low noise level. More details about this deduction are provided in Appendix C.

Fig. 3(c) shows a histogram of the pulse energy of the vibrational Raman line at low power of less than $1 \mathrm{~mW}$, where it approaches a negative exponential distribution. If one compares with the reported experimental results in [19] and [25], this distribution slightly deviates from an ideal exponential distribution, which might be attributed to the influence of the relatively high background noise of the photodetector. A shred of evidence for this deduction is the comparison of the corresponding histogram of the peak intensity in Fig. 3(f), where the distribution is even closer to an exponential distribution due to the less influence of the background noise on the peak intensity when compared to the pulse area. When the laser power increases, the distributions of both pulse energy and peak intensity gradually evolve towards a symmetrical Gaussian-like shape with a narrower width, as shown in Fig. 3(c)-3(e) as well as Fig. 3(f)-3(h). This evolution is consistent with the theoretical prediction and other experimental results of conventional Raman lasers based on a bulk gas cell configuration [19], [25].

\section{B. Long-Term Stability}

As mentioned in the introduction, the generation of one $4.22 \mu \mathrm{m}$ Stokes photon from its $1.53 \mu \mathrm{m}$ pump is accompanied by high energy phonon release. Here, the average heat release from each pump pulse at the quantum efficiency of $\sim 70 \%$ (at the pressure of $15 \mathrm{bar}$ ) is as high as $\sim 25 \mu \mathrm{J}$. In terms of spatial distribution, heat energy is supposed to be mainly concentrated in the output end part of the ARHCF due to the rapid increase of Stokes pulse energy in this region [7]. Therefore, the temperature field is supposed to have a non-uniform distribution along the fiber, consequently leading to a gas circulation inside the laser system. Meanwhile, since Raman lines initiated by quantum noise have a relatively high fluctuation, the heat release from each pump pulse consequently fluctuates also. The combination of gas circulation and fluctuation of the released heat energy could easily result in an irregular variation of the gas-filled ARHCF temperature and thus the Raman gain coefficient, thereby compromising the laser's long-term stability. In order to verify this, we first observed the long-term stability at two different power levels, as shown in Fig. 4(a), where the fluctuation of the pulse peak intensity was monitored over $\sim 30$ minutes by recording 120000 pulses with 14.4 ms average time separation between two adjacent pulses. The long-term stability measurement was implemented after 1 hour of warming-up the laser system, to avoid the coupling efficiency drift and ensure that the system had reached a stable state (see Appendix D). It can be seen that the peak intensity of the Raman laser at the power level of $132.8 \mathrm{~mW}$ exhibits obvious drift (red curve in the bottom, right) when compared to that observed when using less than $1 \mathrm{~mW}$ power (grey curve in the bottom, left), whereas the pump laser fluctuation remains at a low and stable level without obvious drift (curves in the top). Note that a similar drift could be observed in terms of pulse energy, and these phenomena are highly repeatable in our experiment. The Allan deviation, which is a numerical model widely used for the evaluation of a system's stability [26], [27], is introduced here to quantitatively describe the drift effect, as shown in Fig. 4(b), where the Allan deviation of the pulse peak intensities are presented at four average power levels. Initially, because it is white noise-dominated, the Allan deviation linearly decreases with increasing time [26]. When the time is sufficiently long to include the drift effect, the Allan deviation gradually deviates from the linear decrease and finally starts to increase again, i.e., entering the drift-dominant region [26]. The time $t_{\min }$ at the point of inflection is, therefore, a sign of the magnitude of drift. Here $t_{\min }$ is $\sim 106 \mathrm{~s}$ at a Raman laser power of less than $1 \mathrm{~mW}$ and it then gradually decreases to $48 \mathrm{~s}$ when the power increases to $132.8 \mathrm{~mW}$, indicating that obvious drift is induced when increasing the power.

Note that the pump drift maintains a similar level at different powers. Fig. 4(c) presents the Allan deviations of the pump laser corresponding to Fig. 4(b). It can be seen that, although their values at the beginning time have a slight difference, they evolve 

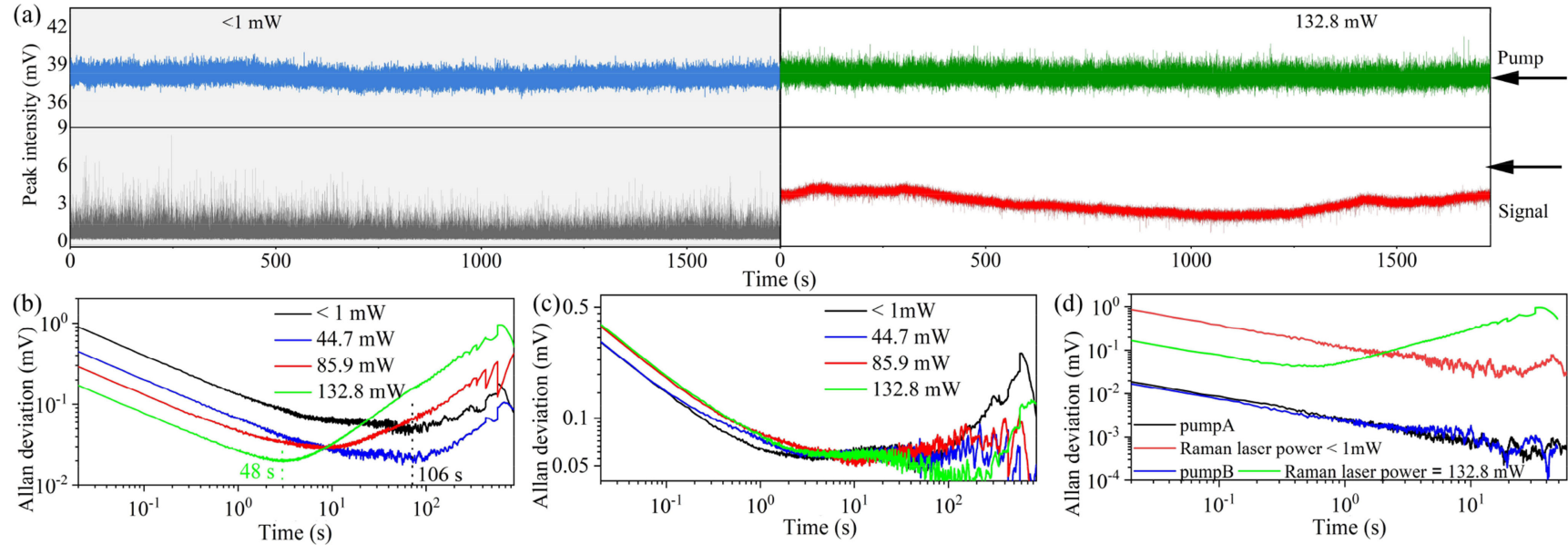

Fig. 4. (a) Long term stability monitoring of the pulse peak intensity of the pump laser (top) and corresponding vibrational Raman laser (bottom) at two different Raman laser powers of $<1 \mathrm{~mW}$ (left) and $132.8 \mathrm{~mW}$ (right). (b) Allan deviation of the vibrational Raman pulse peak intensity at different Raman laser power levels. (c) Allan deviation of the pump pulse peak intensity corresponding to (b). (d) Allan deviations of pump pulse intensity by filtering out their drift parts, as well as the corresponding Allan deviations of the Raman lasers (PumpA and pumpB in (d) donate the pump corresponding to the Raman laser with powers of less than $1 \mathrm{~mW}$ and equal to $132.8 \mathrm{~mW}$, respectively).

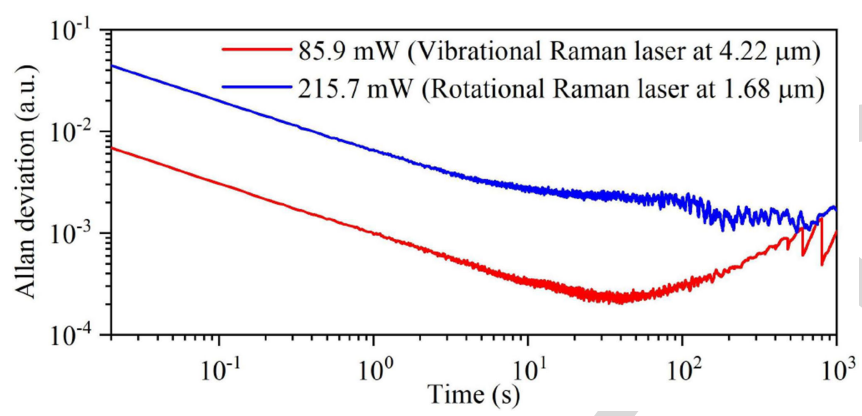

Fig. 5. Comparison of the Allan deviations of the vibrational and rotationa Raman lasers under the same quantum efficiency of $\sim 45 \%$.

with similar trend and gradually overlap with each other. In order to exclude the influence of the pump drift on the drift of the Raman laser, we discarded the pulse pairs where the pump peak intensity falls outside $0.1 \%$ of its average value. Then, the time separation of adjacent measured pulses is reset to $14.4 \mathrm{~ms}$, to calculate the Allan deviation. The pump Allan deviations after the data processing are presented in Fig. 4(d), where their powers are corresponded with the Raman laser powers of $132.8 \mathrm{~mW}$ and $<1 \mathrm{~mW}$, respectively. Both Allan deviations linearly decrease as a function of time, indicating that the drift is effectively mitigated after the data processing. In this case, as shown by the red and green curves in Fig. 4(d), the Allan deviation of Raman laser with $132.8 \mathrm{~mW}$ power still exhibits an obvious inflection point at $\mathrm{t}_{\min }=43 \mathrm{~ms}$, whereas linearly decreases at the power $<1$ $\mathrm{mW}$ as a result of the low amount of heat release. This suggests that the high heat release caused by the high Raman laser energy indeed induces drift of the Raman laser.

The other experimental evidence for the heat-induced drift effect is the comparison of the Allan deviations between the rotational and vibrational Raman lasers, where the Stokes photon at $1.68 \mu \mathrm{m}$ accounts for $\sim 91 \%$ pump photon energy and thus a low heat release. Fig. 5 shows a comparison of the two Allan deviations at the same quantum efficiency of $\sim 45 \%$, i.e., 85.9 $\mathrm{mW}$ for the vibrational Raman laser and $215.7 \mathrm{~mW}$ for the rotational Raman laser. Different from the Allan deviation of vibrational Raman laser, the rotational Raman laser has its Allan deviation continuously decreasing as a function of time. This implies that the drift of the rotational Raman laser here is quite weak due to its small amount of heat release when compared to the vibrational Raman laser.

\section{DisCUSSION AND CONCLUSION}

The noise and drift of the Raman laser could compromise its potential applications such as gas sensing, biology imaging, etc. For instance, in the field of gas monitoring, a long integration or average time is required to offset the influence of the strong noise of the Raman laser, which however slows down the speed of gas detection [26], [28], [29]. Particularly, for some optical sensing methods such as photoacoustic sensing which the detected signal intensity is proportional to the optical pulse energy, the high REN level of the Raman laser (e.g., the minimum REN in this experiment is still up to $16.8 \%$ ) inevitably induces a high noise to the final detected signal [30]. Meanwhile, the detection signal drifts due to the drift of Raman laser, therefore limiting the possibility of noise offset through prolonging integration or average time [26], [31], and finally compromising the detection limit. The Raman laser drift induced by the heat release could be mitigated by avoiding the generation of the phonon, which can be achieved through satisfying the phase matching condition of the generation of anti-Stokes photon [32]. However, it is usually difficult to satisfy the conditions of both phase matching and the efficient emission of mid-IR Raman Stokes with the same fiber parameters and gas pressure. The other possible way to mitigate the Raman laser noise is to suppress the laser fluctuation by reducing the effect of quantum noise by using an external seed. Here, the noise of the $4.22 \mu \mathrm{m}$ vibrational Raman laser could be effectively suppressed by seeding it with a mid-IR quantum 

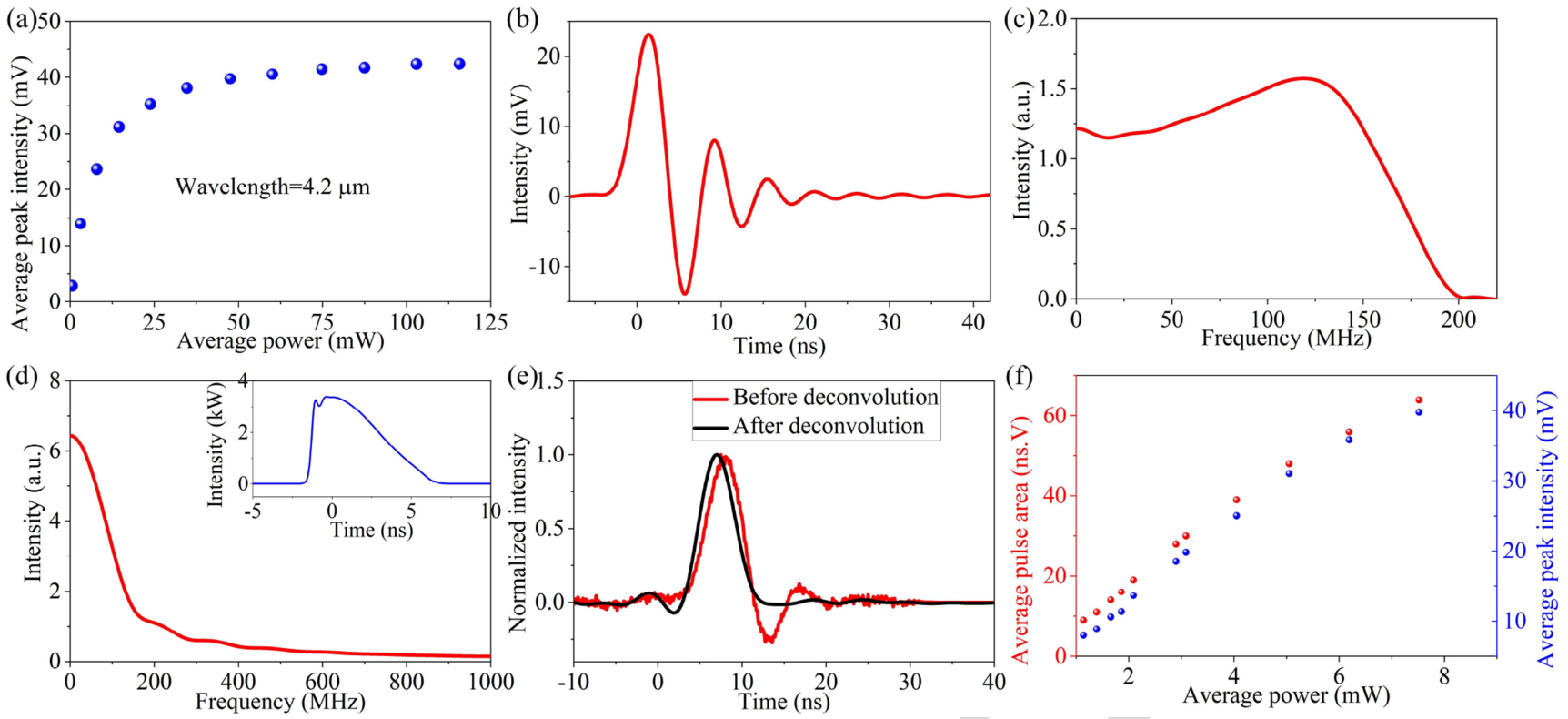

Fig. 6. (a) Output peak intensity of the mid-IR photodetector (PDAVJ10, Thorlabs) as a function of the input power of a $4.2 \mu \mathrm{m}$ femtosecond laser. (b) The impulse function of the photodetector measured in the linear region of (a), with the average over 1000 pulses. (c) Fourier spectrum of the impulse function in (b). (d) The Fourier spectrum of a stimulated Raman laser pulse based on the experimental parameters, where the pump energy is set to a maximum value of $\sim 80 \mu \mathrm{J}$, and $\mathrm{H}_{2}$ pressure to 15 bar. The inset of (d) is the corresponding pulse profile. (e) The measured pulse profile of a $4.22 \mu \mathrm{m}$ Raman laser (red curve), as well as the deconvoluted result (black curve). (f) Evolution of deconvoluted average pulse area and peak intensity as a function of the input power of the $4.22 \mu \mathrm{m}$ Raman laser (The output Raman laser from HCF is fixed at $132 \mathrm{~mW}$, and the power coupled to photodetector is precisely attenuated through a polarizer).

cascade laser which is known for its low-noise level and narrow linewidth.

In conclusion, we investigated the noise and long-term stability of an $\mathrm{H}_{2}$-filled Raman laser in both the near- and mid-IR spectral regions. Both REN and RIN of the presented IR Raman lasers continuously decrease with increasing pulse energy. At the same Raman laser power level, the RIN is always lower than the REN. The mid-IR Raman laser emission is associated with a large amount of heat release and long-term stability monitoring demonstrated some drift in the pulse peak intensity and pulse energy over time. Both the relatively high noise level and long-term drift could be underlying issues for the emerging IR gas-filled Raman lasers, despite their promising advantages of high quantum efficiency and both high energy and the high power laser emission.

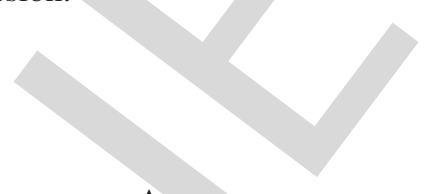

\section{Appendix A}

In this experiment, the measured pulse profile of the $4.22 \mu \mathrm{m}$ Raman laser has a low accuracy because the $\sim 3$ ns rise time of the mid-IR photodetector is comparable with the pulse duration of the Raman laser. Since the photodetector with a much shorter rise time is not commercially available, here we use the deconvolution method to overcome this technical limitation and improve the accuracy of the pulse profile.

It is known that the output signal $O(t)$ from a photodetector is the convolution of the input signal $I(\mathrm{t})$ and the impulse function
$R(t)$ of the photodetector:

$$
O(t)=\int_{-\infty}^{\tau} I(\tau) R(t-\tau) d \tau
$$

We denote the Fourier transformation of $O(t), I(t)$, and $R(t)$ as $\tilde{\mathrm{O}}(\omega), \tilde{\mathrm{I}}(\omega)$, and $\tilde{\mathrm{R}}(\omega)$, respectively. From the convolution theorem, the Fourier transform of $O(t)$ can be expressed as:

$$
\tilde{\mathrm{O}}(\omega)=\tilde{\mathrm{I}}(\omega) \tilde{\mathrm{R}}(\omega)
$$

(1) indicates that $\tilde{\mathrm{I}}(\omega)$ could be calculated by $\tilde{\mathrm{O}}(\omega) / \tilde{\mathrm{R}}(\omega)$, and then $I(t)$ could be retrieved from $\tilde{\mathrm{I}}(\omega)$ through the inverse Fourier transformation.

There are three prerequisites for the accurate retrieving of the $I(t)$ :

1) The impulse function $R(t)$ needs to be known. Here, we use a $\sim 250$ fs linearly polarized laser at $4.22 \mu \mathrm{m}$ to accurately measure the $R(t)$. This femtosecond laser is generated by an optical parametric amplifier (OPA) based on a $10 \mathrm{~mm}$ periodically-poled fan-out $\mathrm{MgO}: \mathrm{LiNbO}_{3}$ crystal. First, the power of the OPA laser is precisely controlled by a polarizer to search the linear region of the photodetector, as shown by Fig. 6 (a). With the increase in average power, it can be seen that the pulse peak intensity slowly increases until it reaches a saturation level. Then, the impulse function is measured by controlling the peak intensity to the center of the linear region, i.e., $\sim 14 \mathrm{mV}$. The measured profile of the impulse function is presented in Fig. 6(b), where 1000 pulses are averaged to smooth the background noise. Fig. 6(c) shows the Fourier transform spectrum of the impulse function in Fig. 6(b), where its maximum frequency $f_{\text {impulse_max }}$ is $\sim 200 \mathrm{MHz}$. 
2) According to the expression of $\tilde{O}(\omega)=\tilde{I}(\omega) \tilde{R}(\omega)$, in order to include the complete information of $\mathrm{I}(\omega)$ into the output signal of $\tilde{\mathrm{O}}(\omega)$, the frequency range of $\tilde{\mathrm{I}}(\omega)$ should be comparable or less than $\tilde{R}(\omega)$. This point is analyzed by comparing the Fourier spectrum of a typical simulated Raman laser pulse profile with the spectrum of $R(\mathrm{t})$. The simulation model is referred to [33], and the parameters are consistent with the experiment setup. The quantum noise model is referred to in [25]. The result is presented in Fig. 6(d), where one can see that the Raman laser pulse (simulated at 15 bar $\mathrm{H}_{2}$ pressure and maximum pump pulse energy of $\sim 80 \mu \mathrm{J})$ and the measured $R(\mathrm{t})$ has a comparable frequency range, thus, indicating that the pulse profile of the Raman laser could be approximately retrieved. Inset of Fig. 6(d) presents the corresponding pulse profile with a pulse duration of $\sim 4.8 \mathrm{~ns}$.

3) Because the output signal from the photodetector is sampled by an oscilloscope, according to the Nyquist sampling theorem, the maximum frequency $\mathrm{f}_{\text {output_max }}$ of the output signal must be less than half of the sampling frequency of the oscilloscope. From the above analysis, the value of $\mathrm{f}_{\text {output_max }}$ must less than $200 \mathrm{MHz}$, whereas the sampling frequency of the oscilloscope is up to $40 \mathrm{GHz}$. Therefore, this condition is well satisfied.

Since the vibrational Raman laser in our experiment approximately satisfies the above all prerequisites, its pulse profile could be basically retrieved. Fig. 6(e) shows a typical comparison of the Raman pulse profiles before and after deconvolution. Compared with the pulse profile before deconvolution with an obvious oscillation at its trailing edge region, the one after deconvolution has a reasonable Gauss-like pulse profile.

The correctness of this method is verified by investigating the evolution of the deconvoluted pulse area and peak intensity as a function of the average Raman laser power, as shown in Fig. 6(f). It should be noted here that the gas-filled Raman laser operates at a high quantum efficiency of $\sim 70 \%$ and pulse energy of $\sim 16 \mu \mathrm{J}$ at the $\mathrm{H}_{2}$ pressure of $15 \mathrm{bar}$, to maximally minimize the noise. The power coupling into the photodetector is precisely attenuated by a polarizer. The measured results show that both the peak intensity and pulse area initially increase in a linear dependence on the input power, and gradually deviates from the linear region because of the saturation effect of the photodetector. This indicates that the noise of both pulse energy and peak power of the Raman laser could be correctly and respectively reflected by the pulse area and peak intensity measured from the oscilloscope, as long as the Raman power is attenuated to the linear region.

\section{Appendix B}

The $4.22 \mu \mathrm{m}$ Raman laser in our experiment shows a high PER of over $-20 \mathrm{~dB}$ because of the use of a linear polarized pump as well as the weak birefringence of the ARHCF. As an example, the measured PER of the Raman laser was up to $-27 \mathrm{~dB}$ and $-22 \mathrm{~dB}$ at average powers of $132.8 \mathrm{~mW}$ and $32.3 \mathrm{~mW}$, respectively, as shown in Fig. 7(a) and 7(b). The PER of the vibrational Raman laser with lower power could
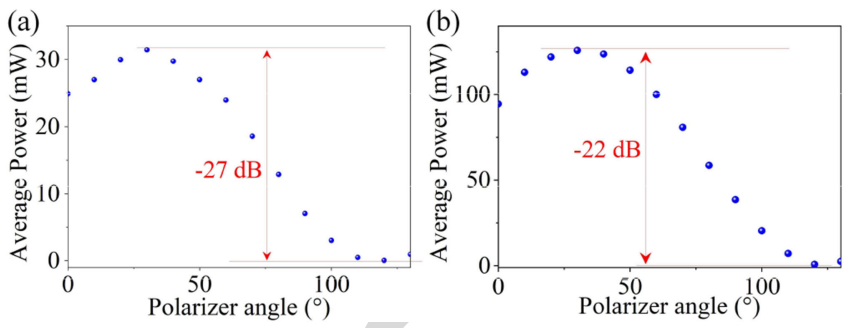

Fig. 7. PER measurement of the $4.22 \mu \mathrm{m}$ Raman laser at the average power of (a) $132.8 \mathrm{~mW}$ and (b) $32.3 \mathrm{~mW}$, respectively.
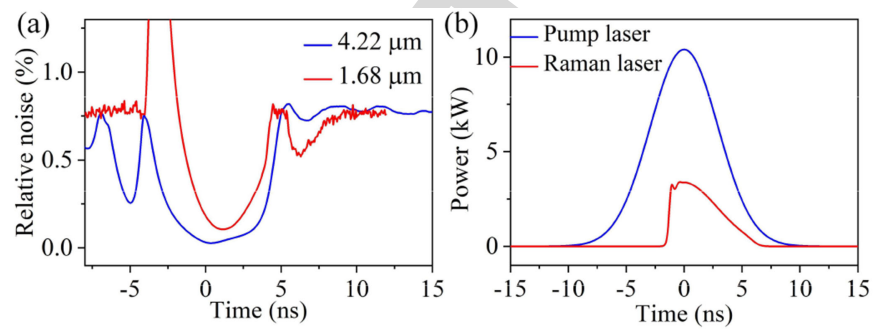

Fig. 8. (a) Instant noise distributions of the $4.22 \mu \mathrm{m}$ vibrational Raman laser and $1.68 \mu \mathrm{m}$ rotational Raman laser from the pulse leading edge to the trailing edge region. The zero time position is the position where the pulse peak power most probably appears. (b) A simulated Raman laser pulse and its corresponding pump pulse at the maximum pump energy of $\sim 80 \mu \mathrm{J}$ and $\mathrm{H}_{2}$ pressure of $15 \mathrm{bar}$.

not be accurately measured since the minimum power after the polarizer approaches the background noise level of the thermal power meter.

\section{Appendix C}

Fig. 8(a) shows the relative noise distributions of the instant pulse intensity from the pulse leading edge to the trailing edge region, for both the $1.68 \mu \mathrm{m}$ rotational Raman laser and $4.22 \mu \mathrm{m}$ vibrational Raman laser, at the same quantum efficiency of $\sim 45 \%$. 10000 pulses are used for each case. The statistics for the $4.22 \mu \mathrm{m}$ Raman laser were computed using the pulses after deconvolution (see Appendix A). The time at zero is the position where the pulse peak most probably appears. It can be seen that the pulse peak region has a lower noise level when compared to the pulse edge regions, indicating that the relative noise of the pulse peak intensity is lower than the pulse energy. This could be attributed to the dependence of the SRS efficiency on the laser intensity, as indicated by the simulation results in Fig. 8(b), where a typical Raman pulse and its corresponding pump pulse are presented for comparison. It can be seen that the peak regions of the Raman laser and pump laser are overlapped, because of the negligible dispersion effect of the gas-filled ARHCF. In this case, the peak region of the Raman pulse has higher SRS efficiency and therefore lower noise level than the pulse edge regions.

\section{Appendix D}

We observed that the coupling loss from the pump laser to the ARHCF can lead to power drift of the coupled pump power due to the heat release. In order to exclude the influence of the coupling efficiency drift during this experiment, the setup was always warming-up over 1 hour before the measurement, so that 

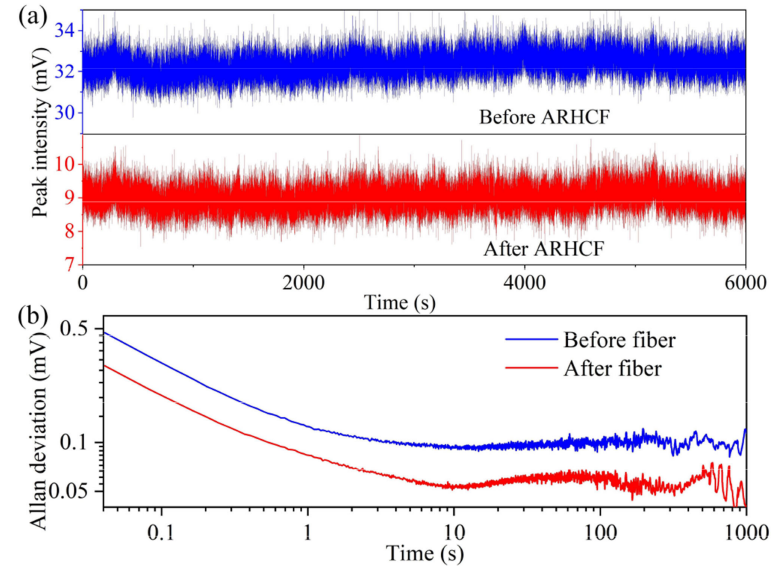

Fig. 9. (a) Long-term stability monitoring of the pump pulse peak intensities before and after the ARHCF by filling ambient air. Pump power was set to the maximum value of $1.4 \mathrm{~W}$. These results were recorded after one hours warmingup of the experiment system. (b) The calculated Allan deviations of the signals in (a).

the heat distribution can reach an equilibrium state. For better clarity, Fig. 9(a) shows a comparison of the long-term monitoring of pump peak intensities before and after the ARHCF. Two near-IR photodetectors (10Gbps PIN-TIA Detector, Nortel, Oclaro; and DET01CFC, Thorlabs) were placed before and after the ARHCF respectively, to simultaneously record the pulse peak intensities. This measurement was implemented by filling ambient air into the ARHCF, to avoid Raman laser-induced noise and drift. The pump laser was set to the maximum power of $1.4 \mathrm{~W}$. The recording started after one-hour warming-up. It can be seen that the signals before and after the ARHCF exhibit a similar fluctuation. This can also be reflected by the calculated Allan deviations in Fig. 9(b), where both curves show a similar evolution tendency as a function of time, and present a common inflection point at $\sim 10 \mathrm{~s}$, indicating that there is no coupling efficiency drift.

\section{ACKNOWLEDGMENT}

The authors would like to thank Callum R. Smith and Shreesha Rao Delanthabettu Shivarama for discussions of noise measurement. The authors also thank Christian R. Petersen for providing the mid-IR OPA.

\section{REFERENCES}

[1] F. Benabid, J. C. Knight, G. Antonopoulos, and P. S. J. Russell, "Stimulated raman scattering in hydrogen-filled hollow-core photonic crystal fiber," Science, vol. 298, no. 5592, pp. 399-402, Oct. 2002.

[2] F. Benabid, F. Couny, J. C. Knight, T. A. Birks, and P. S. J. Russell, "Compact, stable and efficient all-fibre gas cells using hollow-core photonic crystal fibres," Nature, vol. 434, no. 7032, pp. 488-491, Mar. 2005.

[3] C. Markos, J. C. Travers, A. Abdolvand, B. J. Eggleton, and O. Bang, "Hybrid photonic-crystal fiber," Rev. Mod. Phys., vol. 89, no. 4, Nov. 2017, Art. no. 45003.

[4] A. D. Pryamikov, A. S. Biriukov, A. F. Kosolapov, V. G. Plotnichenko, S. L. Semjonov, and E. M. Dianov, "Demonstration of a waveguide regime for a silica hollow - core microstructured optical fiber with a negative curvature of the core boundary in the spectral region $>3.5 \mu \mathrm{m}$," Opt. Exp., vol. 19, no. 2, pp. 1441-1448, Jan. 2011.
[5] M. S. Habib, J. E. Antonio-Lopez, C. Markos, A. Schülzgen, and R. Amezcua-Correa, "Single-mode, low loss hollow-core anti-resonant fiber designs," Opt. Exp., vol. 27, no. 4, Feb. 2019. Art. no. 3824.

[6] M. Klimczak et al., "Nested capillary anti-resonant silica fiber with midinfrared transmission and low bending sensitivity at $4000 \mathrm{~nm}$," Opt. Lett., vol. 44, no. 17, pp. 4395-4398, Aug. 2019.

[7] M. S. Astapovich, A. V. Gladyshev, M. M. Khudyakov, A. F. Kosolapov, M. E. Likhachev, and I. A. Bufetov, "Watt-level nanosecond 4.42- $\mu \mathrm{m}$ Raman laser based on silica fiber," IEEE Photonic. Technol. Lett., vol. 31, no. 1, pp. 78-81, Jan. 2019.

[8] L. Cao, S. Gao, Z. Peng, X. Wang, Y. Wang, and P. Wang, "High peak power $2.8 \mu \mathrm{m}$ Raman laser in a methane-filled negative-curvature fiber," Opt. Exp., vol. 26, no. 5, pp. 5609-5615, Feb. 2018.

[9] A. Gladyshev, Y. Yatsenko, A. Kolyadin, V. Kompanets, and I. Bufetov, "Mid-infrared 10- $\mu \mathrm{J}$-level sub-picosecond pulse generation via stimulated Raman scattering in a gas-filled revolver fiber," Opt. Mater. Exp., vol. 10, no. 12, pp. 3081-3089, Dec. 2020.

[10] A. V. Gladyshev et al., "4.4- $\mu$ m Raman laser based on hollow-core silica fibre," Quantum Electron, vol. 47, no. 5, pp. 491-494, Apr. 2017

[11] Y. Wang et al., "High pulse energy and quantum efficiency mid-infrared gas Raman fiber laser targeting $\mathrm{CO}_{2}$ absorption at $4.2 \mu \mathrm{m}$," Opt. Lett., vol. 45, no. 7, pp. 1938-1941, Mar. 2020.

[12] M. K. Mridha, D. Novoa, S. T. Bauerschmidt, A. Abdolvand, and P. St. J. Russell, "Generation of a vacuum ultraviolet to visible Raman frequency comb in $\mathrm{H}_{2}$-filled kagomé photonic crystal fiber," Opt. Lett., vol. 41, no. 12 , pp. 2811-2814, Jun. 2016.

[13] K. Krupa, K. Baudin, A. Parriaux, G. Fanjoux, and G. Millot, "Intense stimulated Raman scattering in $\mathrm{CO}_{2}$-filled hollow-core fibers," Opt. Lett., vol. 44, no. 21, pp. 5318-5321, Nov. 2019.

[14] Z. Li, W. Huang, Y. Cui, and Z. Wang, "Efficient mid-infrared cascade Raman source in methane-filled hollow-core fibers operating at $2.8 \mu \mathrm{m}$," Opt. Lett., vol. 43, no. 19, pp. 4671-4674, Oct. 2018.

[15] Y. Chen, Z. Wang, B. Gu, F. Yu, and Q. Lu, "Achieving a $1.5 \mu \mathrm{m}$ fiber gas Raman laser source with about $400 \mathrm{~kW}$ of peak power and a $6.3 \mathrm{GHz}$ linewidth," Opt. Lett., vol. 41, no. 21, pp. 5118-5121, Nov. 2016.

[16] M. G. Raymer, I. A. Walmsley, J. Mostowski, and B. Sobolewska, "Quantum theory of spatial and temporal coherence properties of stimulated Raman scattering," Phys. Rev. A, vol. 32, no. 1, pp. 332-344, Jul. 1985.

[17] K. RząŻewski, M. Lewenstein, and M. G. Raymer," "Statistics of stimulated Stokes pulse energies in the steady-state regime," Opt. Commun., vol. 43 , no. 6, pp. 451-454, Nov. 1982.

[18] M. G. Raymer, K. Rzażewski, and J. Mostowski, "Pulse-energy statistics in stimulated Raman scattering," Opt. Lett., vol. 7, no. 2, pp. 71-73, Feb. 1982.

[19] M. G. Raymer and I. A. Walmsley, III The Quantum Coherence Properties of Stimulated Raman scattering. vol. 28, E. B. T.-P. O. Wolf, ed., Amsterdam, The Netherlands; New York: Elsevier, 1990, pp. 181-270.

[20] D. C. MacPherson, R. C. Swanson, and J. L. Carlsten, "Quantum fluctuations in the stimulated-Raman-scattering linewidth," Phys. Rev. Lett. vol. 61, no. 1, pp. 66-69, Jul. 1988.

[21] I. A. Walmsley and M. G. Raymer, "Observation of macroscopic quantum fluctuations in stimulated Raman scattering," Phys. Rev. Lett., vol. 50, no. 13 , pp. 962-965, Mar. 1983

[22] I. A. Walmsley and M. G. Raymer, "Experimental study of the macroscopic quantum fluctuations of partially coherent stimulated Raman scattering," Phys. Rev. A, vol. 33, no. 1, pp. 382-390, Jan. 1986

[23] R. Stolen, "Polarization effects in fiber Raman and Brillouin lasers," IEEE J. Quantum Electron., vol. 15, no. 10, pp. 1157-1160, Oct. 1979.

[24] I. A. Walmsley, M. G. Raymer, T. Sizer, I. N. Duling, and J. D. Kafka, "Stabilization of Stokes pulse energies in the nonlinear regime of stimulated Raman scattering," Opt. Commun., vol. 53, no. 2, pp. 137-140, Feb. 1985.

[25] E. Landahl, D. Baiocchi, and J. R. Thompson, "A simple analytic model for noise shaping by an optical fiber Raman generator," Opt. Commun., vol. 150, no. 1, pp. 339-347, May. 1998.

[26] P. Werle, R. Mücke, and F. Slemr, "The limits of signal averaging in atmospheric trace-gas monitoring by tunable diode-laser absorption spectroscopy (TDLAS)," Appl. Phys. B., vol. 57, no. 2. pp. 131-139, Aug. 1993

[27] D. V. Land, A. P. Levick, and J. W. Hand, "The use of the Allan deviation for the measurement of the noise and drift performance of microwave radiometers," Meas. Sci. Technol., vol. 18, no. 7, pp. 1917-1928, May. 2007.

[28] P. Zhao et al., "Mode-phase-difference photothermal spectroscopy for gas detection with an anti-resonant hollow-core optical fiber," Nat. Commun., vol. 11, no. 1, pp. 1-8, Feb. 2020.

[29] Y. Wang et al., "Mid-infrared photoacoustic gas monitoring driven by a gas-filled hollow-core fiber laser," Sci. Rep., vol. 11, no. 1, pp. 1-8, Feb. 2021. 
[30] T. Schmid, "Photoacoustic spectroscopy for process analysis," Anal. Bioanal. Chem., vol. 384, no. 5, pp. 1071-1086, Jun. 2006.

[31] J. Hodgkinson, R. Smith, W. O. Ho, J. R. Saffell, and R. P. Tatam, "Nondispersive infra-red (NDIR) measurement of carbon dioxide at $4.2 \mu \mathrm{m}$ in a compact and optically efficient sensor," Sensors Actuators B Chem, vol. 186, pp. 580-588, Sep. 2013.

[32] N. Vermeulen, C. Debaes, P. Muys, and H. Thienpont, "Mitigating heat dissipation in Raman lasers using coherent anti-Stokes Raman scattering,' Phys. Rev. Lett., vol. 99, no. 9, Aug. 2007, Art. no. 93903

[33] G. Hilfer and C. R. Menyuk, "Stimulated Raman scattering in the transient limit," J. Opt. Soc. Am. B, vol. 7, no. 5, pp. 739-749, May. 1990.

Yazhou Wang received the B.Sc. degree in physics from Southwest University, the M.Sc. degree in 3D displaying from Sichuan University, and the Ph.D. degree in nonlinear fiber optics from the University of Electronic Science and Technology of China. During the Ph.D., he was a Visiting Student with the Technical University of Denmark in 2018, with the study on air plasma based $\mathrm{THz}$ generation. He received the Ph.D. degree in 2019, he joined Technical University of Denmark as a Post-Doc. His currently study focuses on infrared gas-filled fiber Raman laser design and applications in gas detection. He is a member of OSA and SPIE society.

Abubakar Isa Adamu (Member, IEEE) received the B.Sc. degree in engineering physics from Gaziantep University, Turkey and the M.Sc. degree in material science and nanotechnology from Bilkent University, Turkey, where he worked on development of artificial nose, for sensing of toxic gases with National Nanotechnology Research Centre (UNAM), Turkey. He was a Visiting Student with the University of Chicago, USA, in 2013, where he learnt therapeutic modalities in radiation oncology. He was a Guest Research Student with the University of Twente, The Netherlands, in 2015, where he worked on membranes for next-generation EUV lithography. Currently, he is a Manufacturing Engineer with Lumenisity Ltd, U.K.

He received the Doctorate degree in 2020 from the Technical University of Denmark in photonics engineering, where we worked on hollow-core optical fibres, ultrafast nonlinear optics, supercontinuum laser and fabrication of hybrid speciality fibres. Dr. Adamu is a member of OSA, EPS, and SPIE. He was recently awarded the Outstanding Student Paper Award at SPIE Photonics West, 2019, San Francisco, USA and Best Research Poster Award by Danish Optical Society (DOPS).

Manoj K. Dasa was born in Hyderabad, India, in 1991. He received the B.Tech degree in electronics and communications engineering from Jawaharlal Nehru Technological University, Hyderabad, India, and the M.Sc. degree in electrical communications engineering with a specialization in optoelectronics from the University of Kassel, Germany, in April 2016. In February 2017, he joined the Technical University of Denmark as a Marie Curie Ph.D. Fellow with the Department of Photonics Engineering. His Ph.D. degree focused on developing high-pulse energy supercontinuum sources for multispectral photoacoustic applications in the extended near-infrared region. His research interest includes supercontinuum lasers, biomedical imaging, and spectroscopy.

Jose E. Antonio-Lopez received the Ph.D. degree from the Instituto Nacional de Astrofísica, Óptica y Electrónica (INAOE), San Andres Cholula, Mexico, in 2012, with the work design and fabrication of photonic devices based on multimode interference. He is a Research Scientist with the College of Optics and Photonics (CREOL), University of Central Florida (UCF), Orlando, FL, USA. His research interests include design, fabrication, and use of special fibers
Md. Selim Habib (Senior Member, IEEE) received the B.Sc. and M.Sc. degrees in electrical and electronic engineering from the Rajshahi University of Engineering and Technology, Rajshahi, Bangladesh, in 2008 and 2012, respectively. He received the Ph.D. degree from Technical University of Denmark (DTU) in 2017. After finishing his Ph.D., he joined as a Postdoctoral Researcher in Fibers Sensors and Supercontinuum Group with the Department of Photonics Engineering, DTU. After finishing his Postdoctoral Fellowship with DTU, he worked as a Postdoctoral Research Associate with CREOL, The College of Optics and Photonics, University of Central Florida, USA, from 2017 to 2019. $\mathrm{He}$ is currently an Assistant Professor of Electrical and Computer Engineering with Florida Polytechnic University, USA. He has authored or coauthored more than 50 articles in referred journals. His research mainly focuses on design, fabrication, and characterization of low loss hollow-core fiber in the near-IR to mid-IR, light gas nonlinear interaction in hollow-core fibers, supercontinuum generation, and multi-mode nonlinear optics.

Dr. Habib is a Senior Member of Institute of Electrical and Electronics Engineers (IEEE), Optical Society of America (OSA) Early Careers Member, and Executive officer of OSA Fiber modeling and Fabrication group. Dr. Habib is an Associate Editor of IEEE ACCESS, and Feature Editor of Applied Optics (OSA). He was the recipient of the University Gold Medal Award from Rajshahi University of Engineering and Technology in 2014.

Rodrigo Amezcua Correa received the Ph.D. degree from the Optoelectronics Research Centre University of Southampton, Southampton, U.K. Since 2011, he has been with CREOL, The College of Optics and Photonics, University of Central Florida, Orlando, FL, USA. He is currently an Associate Professor of optics and photonics with CREOL. His research interests include optical fibers and fiber devices, fiber laser systems, fiber optic sensing, and optical communications.

Ole Bang received the M.Sc. degree in electrical engineering from the Technical University of Denmark (DTU), Kongens Lyngby, Denmark, in 1992 and the $\mathrm{Ph} . \mathrm{D}$. degree in nonlinear physics from DTU in 1993. From 1993 to 1995, he was a Postdoc with the Laboratoire de Physique, Ecole Normale Supérieure de Lyon, Lyon, France, working on discrete physical models of the nonlinear dynamics of biomolecules. From 1995 to 1999, he was a Research Fellow with Optical Sciences Centre, Australian National University, Canberra, ACT, Australia, where he worked theoretically on nonlinear optics, in particular, on solitons and modulational instability in materials with a quadratic nonlinearity and quasi-phase-matching gratings. From 1999 to 2003, he was an Associate Professor with the Department of Informatics and Mathematical Modeling, DTU. Since 2003, he has been with DTU Fotonik, Department of Photonics Engineering, DTU, where he became Professor in 2011, and is currently heading the Fiber Sensors \& Supercontinuum group. His current research interests include microstructured optical fibers in silica, polymer, and soft glasses, fiber optical sensors for sensing biomolecules, $\mathrm{pH}$, Oxygen, humidity, stress/strain, in particular using Fiber Bragg gratings, and the fabrication and application of supercontinuum sources, in particular in spectroscopy and imaging, such as OCT and optical-resolution photo-acoustic microscopy. He has been a consultant in supercontinuum sources with NKT Photonics since 2009. In 2015, he established the company SHUTE Sensing Solutions to market polymer fiber sensors and in 2018, he established the company NORBLIS to market mid-IR supercontinuum lasers and OCT systems.

Christos Markos received the B.Eng. (Hons) and M.Sc. (with Distinction) degree from the University of Liverpool in Electrical Engineering and Electronics, in 2007 and 2008. He continued his studies as Ph.D. student with the National Hellenic Research Foundation, Theoretical and Physical Chemistry Institute, Athens and received the Ph.D. degree in optics/optoelectronics in 2013. His main research activities are broad within the field of optical materials and nonlinear optics and particularly in gas-filled hollow-core fiber lasers, chalcogenide glasses, imaging and multimaterial fiber optics towards development of novel neural interfaces and optoelectronic smart systems. He has joined and worked with several distinguished research groups in USA and Europe including the Multi-material Optical Fiber Devices Group in the College of Optics and Photonics (CREOL), USA and Mid-Infrared Photonics Group with the University of Nottingham, U.K. He has established three state-of-the-art laboratories at DTU Fotonik for soft-glass synthesis, extrusion and fiber fabrication and since 2018 holds the position of Associate Professor. He is a member of OSA and SPIE society, Associate Editor of Optical Material Express journal and co-founder of NORBLIS. 


\section{1}

\title{
Noise Performance and Long-Term Stability of Near- and Mid-IR Gas-Filled Fiber Raman Lasers
}

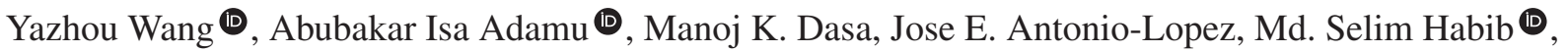 \\ Rodrigo Amezcua-Correa, Ole Bang, and Christos Markos (1)
}

\begin{abstract}
Stimulated Raman scattering (SRS) enabled by the emerging gas-filled low-loss anti-resonant hollow-core fiber (ARHCF) technology opens up a competitive way towards the development of novel lasers in the molecular fingerprint region. In this article, the characteristics of noise and long-term stability of near- and mid-infrared (near-IR and mid-IR) gas-filled fiber Raman lasers have been investigated for the first time. The results reveal that an increase in Raman pulse energy is associated with a decrease in noise, and that the relative pulse peak intensity noise (RIN) is always lower than the relative pulse energy noise (REN). We also demonstrate that long-term drift of the pulse energy and peak power are directly linked with the high amount of heat release during the Raman Stokes generation. The demonstrated noise and long-term stability performance provide necessary references for potential spectroscopic applications as well as further improvements of the emerging IR gas-filled ARHCF Raman laser technology.
\end{abstract}

Index Terms-Laser noise, laser stability, optical fiber lasers, Raman lasers.

Manuscript received January 4, 2021; revised March 3, 2021; accepted March 11, 2021. This work was supported in part by Danmarks Frie Forskningsfond Hi-SPEC under the Grant 8022-00091B, Lundbeck Foundation under MultiBRAIN project (R276-2018-869), VILLUM FONDEN under Grant 36063, Innovation Fund Denmark UVSUPER under Grant 8090-00060A, ECOMETA under the Grant 6150-00030B, and in part by U.S. ARO under Grant W911NF19-1-0426. (Corresponding authors: Yazhou Wang; Christos Markos.)

Yazhou Wang, Abubakar Isa Adamu, and Manoj K. Dasa are with the Department of Photonics Engineering, Technical University of Denmark, DK- 2800 Lyngby, Denmark (e-mail: yazwang@ fotonik.dtu.dk; abisa@ fotonik.dtu.dk; manda@fotonik.dtu.dk).

Ole Bang is with the Department of Photonics Engineering, Technical University of Denmark, DK- 2800 Lyngby, Denmark, and with NKT Photonics, 3460 Birkerød, Denmark, and with the NORBLIS IVS, 2830 Virum, Denmark (e-mail: oban@fotonik.dtu.dk).

Christos Markos is with the Department of Photonics Engineering, Technical University of Denmark, DK- 2800 Lyngby, Denmark, and also with the NORBLIS IVS, 2830 Virum, Denmark (e-mail: chmar@ fotonik.dtu.dk).

Jose E. Antonio-Lopez and Rodrigo Amezcua-Correa are with the College of Optics and Photonics, University of Central Florida, Orlando FL-32816 USA (e-mail: jealopez@creol.ucf.edu; r.amezcua@creol.ucf.edu).

Md. Selim Habib is with the Department of Electrical and Computer Engineering, Florida Polytechnic University, Lakeland FL-33805 USA (e-mail: mhabib@floridapoly.edu).

Color versions of one or more figures in this article are available at https: //doi.org/10.1109/JLT.2021.3067514.

Digital Object Identifier 10.1109/JLT.2021.3067514 its compelling features that enable efficient stimulated Raman scattering (SRS) process, high damage threshold, broad transmission range, compact fiber structure, high freedom on wavelength selection, etc. [1]-[3]. Especially, with the recent advent of low loss anti-resonant HCF (ARHCF) technology [4]-[6], the laser wavelength has been extended to the mid-infrared (mid-IR) region where the high silica loss could be significantly mitigated by confining most power of the laser beam within the fiber core (gas) region [7]-[9]. A series of gas-filled fiber Raman lasers have been reported from the UV to mid-IR region [7], [10]-[15]. Despite their unique advantages compared to other counterparts, high noise is an intrinsic issue of Raman lasers since the SRS process is usually initiated by the quantum noise [16]-[18]. The noise of Raman laser has extensively been investigated in the frame of a bulk gas cell usually filled with hydrogen $\left(\mathrm{H}_{2}\right)$ [19]-[22], but yet remains unexplored for the gas-filled fiber technology. The noise characteristics of the latter is worth further investigation since the SRS process in gas-filled HCF is much more efficient than the conventional bulk gas cell structure. Besides, for the Raman Stokes emission involving a large photon energy difference between the pump and Stokes, the energy of the released phonon is high and thus could easily lead to a large amount of heat release within the core region of the HCF, where the gas circulation speed is much slower than its counterpart of using bulk gas cell. As a result, the temperature and therefore the Raman gain coefficient will be directly affected. In the last few years, several efficient Raman lasers operating at $\sim 4 \mu \mathrm{m}$ wavelength have been reported with $\mathrm{H}_{2}$-filled ARHCFs by using $1.5 \mu \mathrm{m}$ fiber laser as a pump [7], [10], [11], where the generation of one Stokes photon is accompanied by phonon emission with up to $\sim 60 \%$ of pump photon energy. Consequently, the heat pulse energy of these mid-IR Raman lasers is calculated to be $\mu \mathrm{J}$ level, which could impose a significant impact on the laser stability. However, as an emerging laser technology, these issues have not been investigated yet.

In this work, we thoroughly investigated the noise and stability performance of two different Raman lasers based on an $\mathrm{H}_{2}$-filled ARHCF, at $1.68 \mu \mathrm{m}$ and $4.2 \mu \mathrm{m}$. The Raman lasers were generated by pumping with a custom-made linearly polarized fiber laser at $1532.8 \mathrm{~nm}$. The pump laser has a pulse energy of $\sim 80 \mu \mathrm{J}$, pulse duration of $7 \mathrm{~ns}$, repetition rate of $8 \mathrm{kHz}$, and linewidth of $\sim 0.06 \mathrm{~nm}$. The ARHCF is designed with two transmission windows around $1.3-1.8 \mu \mathrm{m}$, and $2.5-4.5 \mu \mathrm{m}$, separated by a resonance peak at $\sim 2 \mu \mathrm{m}[11]$. 


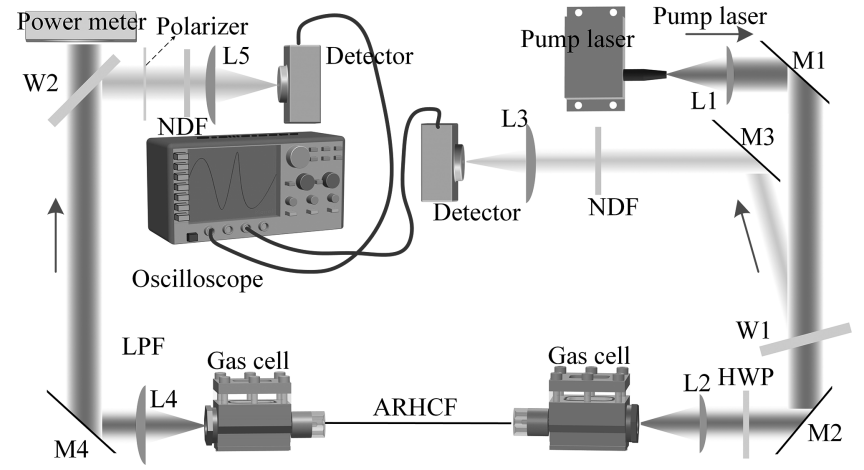

Fig. 1. Noise measurement setup of gas-filled fiber Raman laser. M1-M4 are silver mirrors. L1-L3 are C-coated plano-convex silica lenses, L4-L5 are uncoated plano-convex $\mathrm{CaF}_{2}$ lenses. $\mathrm{W} 1$ and $\mathrm{W} 2$ are respectively optical wedge and window used to extract a small part of the laser beam for pulse detection with photodetectors.

\section{EXPERIMENT SETUP AND METHOD}

Fig. 1 shows the details of the experimental setup used for this investigation. $\mathrm{H}_{2}$ is filled into the ARHCF through two gas cells. At the pressure range of 5-20 bar, $\mathrm{H}_{2}$ inside the ARHCF quickly reaches to equilibrium state within one minute. The fiber length was optimized to $3.95 \mathrm{~m}$, to obtain a maximum

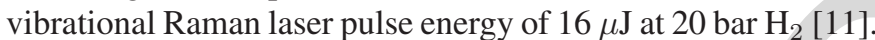
The output from the $\mathrm{H}_{2}$-filled ARHCF is first collimated and then the residual pump is removed by a long-pass filter (LPF). The remaining $4.22 \mu \mathrm{m}$ Raman part is passed through a $\mathrm{CaF}_{2}$ window ( $\sim 95 \%$ transmission at $4.22 \mu \mathrm{m})$ and monitored by a thermal power meter. A small part of the Raman laser reflected from the front surface of the $\mathrm{CaF}_{2}$ window is focused onto a photodetector (PDAVJ10, Thorlabs) which is connected to an oscilloscope (Teledyne Lecroy HD09000) for pulse profile monitoring. The pulse duration of the Raman laser is estimated to be a few nanoseconds, which is comparable with the rise time of the photodetector $(\sim 3.5 \mathrm{~ns})$, as a result, the Raman laser pulse profile couldn't be accurately measured. Here we use the deconvolution method to retrieve the original pulse profile, as described in Appendix A. Based on this method, we found that both pulse energy and peak power of the Raman laser could be correctly and respectively reflected by the pulse area and peak intensity measured from the oscilloscope, as long as the Raman power is attenuated to the linear region of the photodetector. Because the $4.22 \mu \mathrm{m}$ Raman line here has a high polarization extinction ratio (PER) $(>20 \mathrm{~dB}$ ) (see Appendix B), a polarizer was used to continuously attenuate the average power, so that most of the pulses could be appropriately measured in the linear response region of the photodetector. The characterization of the photodetector is provided in Appendix A. The pump laser was also simultaneously monitored by focusing a minor part of the pump into a near-infrared (near-IR) photodetector (DET01CFC, Thorlabs).

From the setup in Fig. 1, due to the fact that the pump is linearly polarized, the rotational Raman line at $1.68 \mu \mathrm{m}$ has a near-zero power (see the measured spectrum in Fig. 2) and thus negligible influence on the generation of a vibrational Raman line at $4.22 \mu \mathrm{m}$. Nevertheless, for the purpose of comparison,

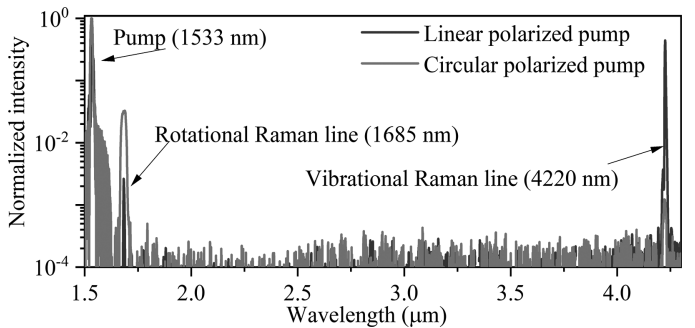

Fig. 2. Spectra comparison of the Raman lines with linear and circular polarized pumps.

we also monitored the noise and long-term stability of the rotational Raman line generated at $1.68 \mu \mathrm{m}$ when replacing the half-wave plate (HWP) with a quarter-wave plate (QWP) in front of the ARHCF to make the pump light circularly polarized [23]. When using circularly polarized pump light, the $\mathrm{H}_{2}$ pressure was reduced to 8 bar to suppress the vibrational Raman line, which finally provided a quantum efficiency and pulse energy of the rotational Raman line of $45 \%$ and $27 \mu \mathrm{J}$, respectively, whereas the average power of the vibrational Raman line was measured to be less than $1 \mathrm{~mW}$ (see Fig. 2). Then, the pulse profile of the rotational Raman line was precisely measured by the other near-IR photodetector with the same parameters as the one used for monitoring the pump. Because of the circular polarization of the rotational Raman line, the polarizer was replaced by a continuously variable near-IR neutral density filter (NDF) (NDL-10C-2, Thorlabs), to precisely attenuate the pulse peak power.

\section{RESULTS AND ANALYSIS}

\section{A. Noise Performance}

In the noise measurement, we collected a train of 10000 Raman pulses and corresponding pump pulses, for each pump power level. The influence of the pump noise is suppressed by discarding the pump pulses whose peak intensity falls outside $2 \%$ of its average value. After the data processing, the number of pairs is reduced to $\sim 9500$, and the relative noise (defined as the ratio of the standard deviation to the mean value) of the pump peak intensity is reduced from $\sim 1.7 \%$ to $\sim 1 \%$. Fig. 3(a) and 3(b) present the measured relative pulse energy noise (REN) and relative pulse peak intensity noise (RIN) of the vibrational and rotational Raman lasers. It shows that both of them gradually decrease, and in Fig. 3(a) they finally approach a stable level as the Raman laser power exceeds $\sim 80 \mathrm{~mW}$ (corresponding to $\sim 45 \%$ quantum efficiency). This is attributed to the stabilization effect of Stokes pulse energy induced by the depletion of pump power as well as $\mathrm{H}_{2}$ molecules in the ground state during the SRS [24]. Meanwhile, at the same pulse energy level, it can be seen that the RIN is always lower than the REN. For instance, at the highest power of $132.8 \mathrm{~mW}$ in Fig. 3(a), the REN is $16.8 \%$, whereas the RIN is as low as $2.9 \%$. This difference is supposed to be caused by the dependence of the SRS efficiency on the light intensity: the SRS process in the peak region of the Raman pulse is more efficient than at its edge regions, whereas high SRS 

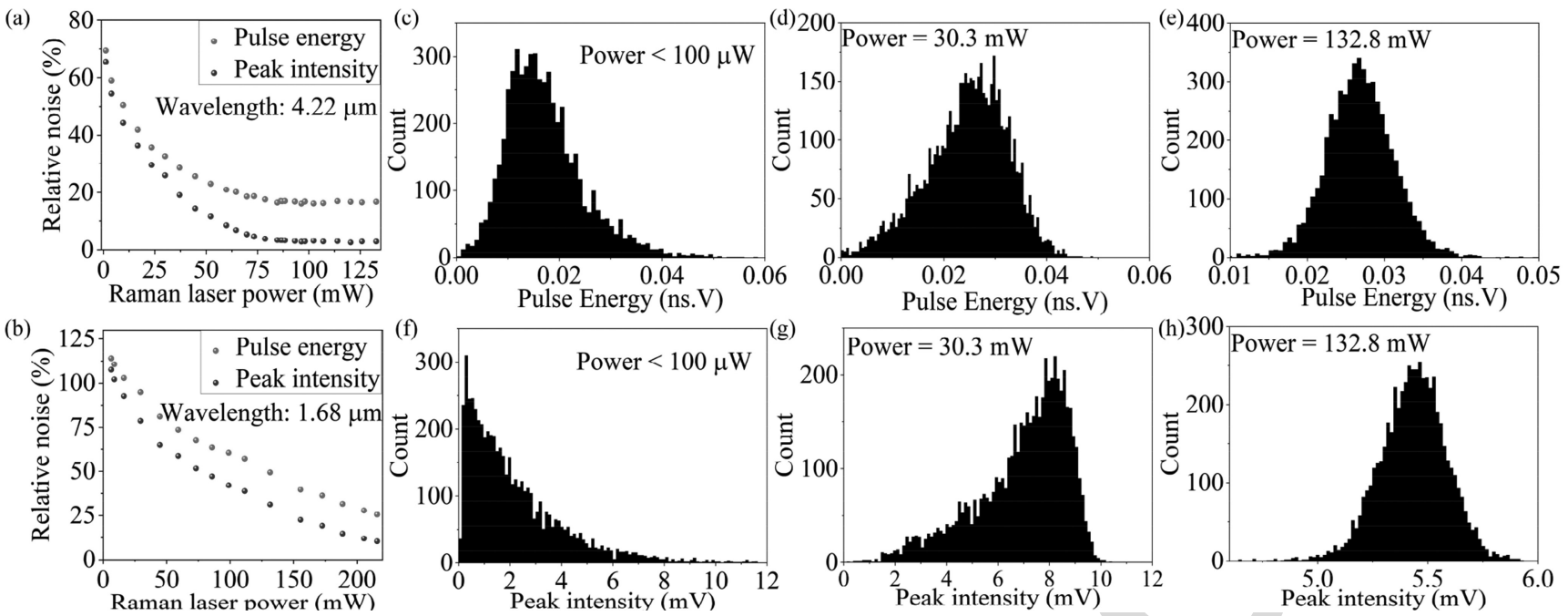

Fig. 3. (a) and (b) are the measured REN and RIN of the $4.22 \mu \mathrm{m}$ and $1.68 \mu \mathrm{m}$ Raman lines as a function of their average powers, respectively. (c)-(e) are histograms of measured pulse energy at different levels of output Raman laser power (4.22 $\mu \mathrm{m})$. (f)-(h) are histograms of measured pulse peak intensity corresponding to (c)-(e).

efficiency is associated with the low noise level. More details about this deduction are provided in Appendix C.

Fig. 3(c) shows a histogram of the pulse energy of the vibrational Raman line at low power of less than $1 \mathrm{~mW}$, where it approaches a negative exponential distribution. If one compares with the reported experimental results in [19] and [25], this distribution slightly deviates from an ideal exponential distribution, which might be attributed to the influence of the relatively high background noise of the photodetector. A shred of evidence for this deduction is the comparison of the corresponding histogram of the peak intensity in Fig. 3(f), where the distribution is even closer to an exponential distribution due to the less influence of the background noise on the peak intensity when compared to the pulse area. When the laser power increases, the distributions of both pulse energy and peak intensity gradually evolve towards a symmetrical Gaussian-like shape with a narrower width, as shown in Fig. 3(c)-3(e) as well as Fig. 3(f)-3(h). This evolution is consistent with the theoretical prediction and other experimental results of conventional Raman lasers based on a bulk gas cell configuration [19], [25].

\section{B. Long-Term Stability}

As mentioned in the introduction, the generation of one $4.22 \mu \mathrm{m}$ Stokes photon from its $1.53 \mu \mathrm{m}$ pump is accompanied by high energy phonon release. Here, the average heat release from each pump pulse at the quantum efficiency of $\sim 70 \%$ (at the pressure of $15 \mathrm{bar}$ ) is as high as $\sim 25 \mu \mathrm{J}$. In terms of spatial distribution, heat energy is supposed to be mainly concentrated in the output end part of the ARHCF due to the rapid increase of Stokes pulse energy in this region [7]. Therefore, the temperature field is supposed to have a non-uniform distribution along the fiber, consequently leading to a gas circulation inside the laser system. Meanwhile, since Raman lines initiated by quantum noise have a relatively high fluctuation, the heat release from each pump pulse consequently fluctuates also. The combination of gas circulation and fluctuation of the released heat energy could easily result in an irregular variation of the gas-filled ARHCF temperature and thus the Raman gain coefficient, thereby compromising the laser's long-term stability. In order to verify this, we first observed the long-term stability at two different power levels, as shown in Fig. 4(a), where the fluctuation of the pulse peak intensity was monitored over $\sim 30$ minutes by recording 120000 pulses with 14.4 ms average time separation between two adjacent pulses. The long-term stability measurement was implemented after 1 hour of warming-up the laser system, to avoid the coupling efficiency drift and ensure that the system had reached a stable state (see Appendix D). It can be seen that the peak intensity of the Raman laser at the power level of $132.8 \mathrm{~mW}$ exhibits obvious drift (red curve in the bottom, right) when compared to that observed when using less than $1 \mathrm{~mW}$ power (grey curve in the bottom, left), whereas the pump laser fluctuation remains at a low and stable level without obvious drift (curves in the top). Note that a similar drift could be observed in terms of pulse energy, and these phenomena are highly repeatable in our experiment. The Allan deviation, which is a numerical model widely used for the evaluation of a system's stability [26], [27], is introduced here to quantitatively describe the drift effect, as shown in Fig. 4(b), where the Allan deviation of the pulse peak intensities are presented at four average power levels. Initially, because it is white noise-dominated, the Allan deviation linearly decreases with increasing time [26]. When the time is sufficiently long to include the drift effect, the Allan deviation gradually deviates from the linear decrease and finally starts to increase again, i.e., entering the drift-dominant region [26]. The time $t_{\min }$ at the point of inflection is, therefore, a sign of the magnitude of drift. Here $t_{\min }$ is $\sim 106 \mathrm{~s}$ at a Raman laser power of less than $1 \mathrm{~mW}$ and it then gradually decreases to $48 \mathrm{~s}$ when the power increases to $132.8 \mathrm{~mW}$, indicating that obvious drift is induced when increasing the power.

Note that the pump drift maintains a similar level at different powers. Fig. 4(c) presents the Allan deviations of the pump laser corresponding to Fig. 4(b). It can be seen that, although their values at the beginning time have a slight difference, they evolve 

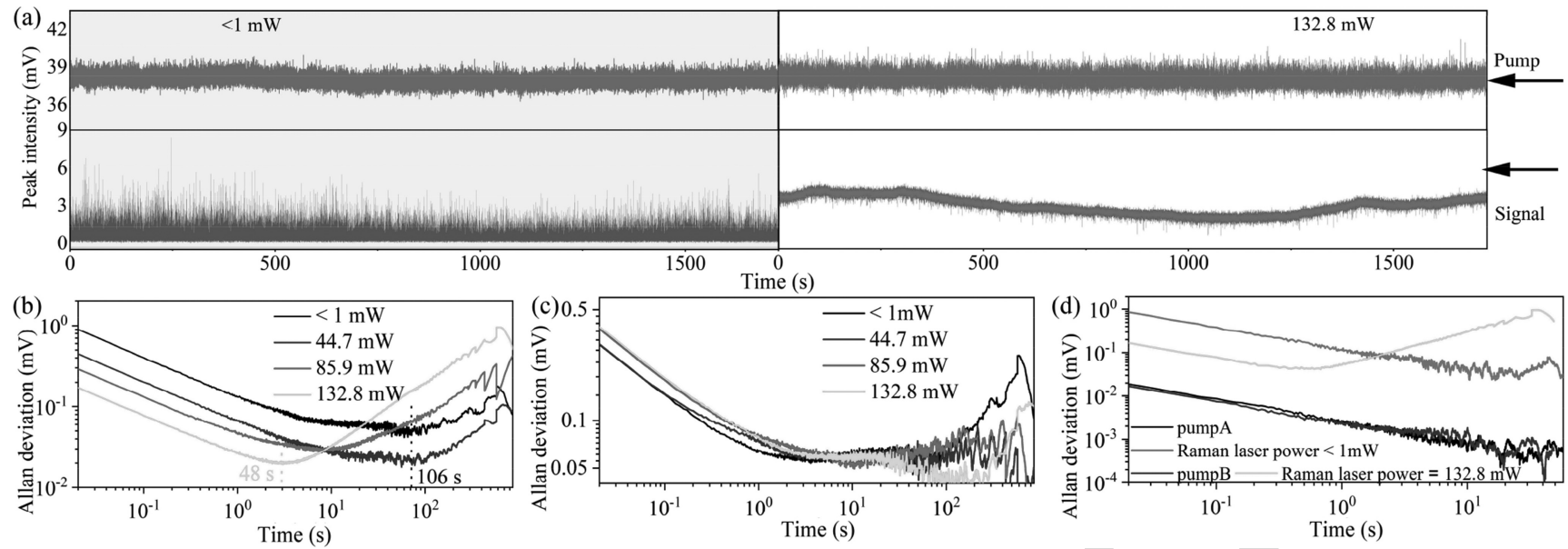

Fig. 4. (a) Long term stability monitoring of the pulse peak intensity of the pump laser (top) and corresponding vibrational Raman laser (bottom) at two different Raman laser powers of $<1 \mathrm{~mW}$ (left) and $132.8 \mathrm{~mW}$ (right). (b) Allan deviation of the vibrational Raman pulse peak intensity at different Raman laser power levels. (c) Allan deviation of the pump pulse peak intensity corresponding to (b). (d) Allan deviations of pump pulse intensity by filtering out their drift parts, as well as the corresponding Allan deviations of the Raman lasers (PumpA and pumpB in (d) donate the pump corresponding to the Raman laser with powers of less than $1 \mathrm{~mW}$ and equal to $132.8 \mathrm{~mW}$, respectively).

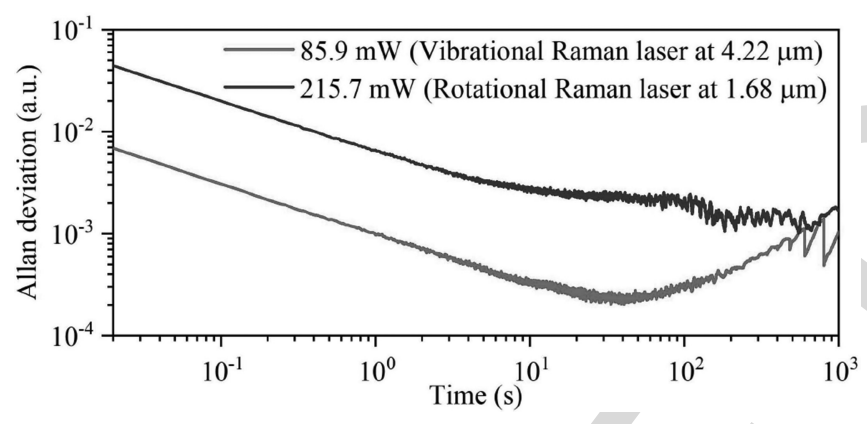

Fig. 5. Comparison of the Allan deviations of the vibrational and rotational Raman lasers under the same quantum efficiency of $\sim 45 \%$. with similar trend and gradually overlap with each other. In order to exclude the influence of the pump drift on the drift of the Raman laser, we discarded the pulse pairs where the pump peak intensity falls outside $0.1 \%$ of its average value. Then, the time separation of adjacent measured pulses is reset to $14.4 \mathrm{~ms}$, to calculate the Allan deviation. The pump Allan deviations after the data processing are presented in Fig. 4(d), where their powers are corresponded with the Raman laser powers of $132.8 \mathrm{~mW}$ and $<1 \mathrm{~mW}$, respectively. Both Allan deviations linearly decrease as a function of time, indicating that the drift is effectively mitigated after the data processing. In this case, as shown by the red and green curves in Fig. 4(d), the Allan deviation of Raman laser with $132.8 \mathrm{~mW}$ power still exhibits an obvious inflection point at $\mathrm{t}_{\min }=43 \mathrm{~ms}$, whereas linearly decreases at the power $<1$ $\mathrm{mW}$ as a result of the low amount of heat release. This suggests that the high heat release caused by the high Raman laser energy indeed induces drift of the Raman laser.

The other experimental evidence for the heat-induced drift effect is the comparison of the Allan deviations between the rotational and vibrational Raman lasers, where the Stokes photon at $1.68 \mu \mathrm{m}$ accounts for $\sim 91 \%$ pump photon energy and thus a low heat release. Fig. 5 shows a comparison of the two Allan deviations at the same quantum efficiency of $\sim 45 \%$, i.e., 85.9 $\mathrm{mW}$ for the vibrational Raman laser and $215.7 \mathrm{~mW}$ for the rotational Raman laser. Different from the Allan deviation of vibrational Raman laser, the rotational Raman laser has its Allan deviation continuously decreasing as a function of time. This implies that the drift of the rotational Raman laser here is quite weak due to its small amount of heat release when compared to the vibrational Raman laser.

\section{DisCUSSION AND CONCLUSION}

The noise and drift of the Raman laser could compromise its potential applications such as gas sensing, biology imaging, etc. For instance, in the field of gas monitoring, a long integration or average time is required to offset the influence of the strong noise of the Raman laser, which however slows down the speed of gas detection [26], [28], [29]. Particularly, for some optical sensing methods such as photoacoustic sensing which the detected signal intensity is proportional to the optical pulse energy, the high REN level of the Raman laser (e.g., the minimum REN in this experiment is still up to $16.8 \%$ ) inevitably induces a high noise to the final detected signal [30]. Meanwhile, the detection signal drifts due to the drift of Raman laser, therefore limiting the possibility of noise offset through prolonging integration or average time [26], [31], and finally compromising the detection limit. The Raman laser drift induced by the heat release could be mitigated by avoiding the generation of the phonon, which can be achieved through satisfying the phase matching condition of the generation of anti-Stokes photon [32]. However, it is usually difficult to satisfy the conditions of both phase matching and the efficient emission of mid-IR Raman Stokes with the same fiber parameters and gas pressure. The other possible way to mitigate the Raman laser noise is to suppress the laser fluctuation by reducing the effect of quantum noise by using an external seed. Here, the noise of the $4.22 \mu \mathrm{m}$ vibrational Raman laser could be effectively suppressed by seeding it with a mid-IR quantum 

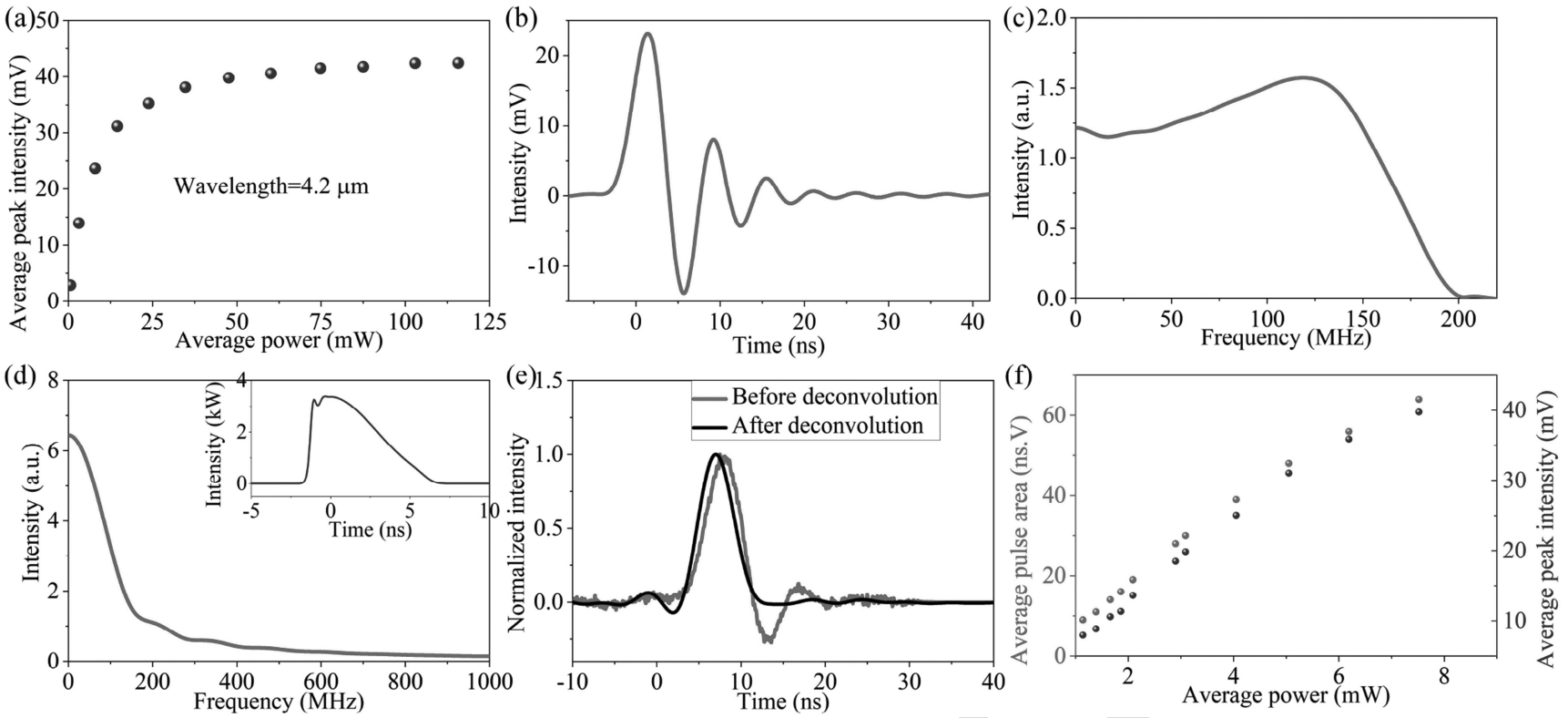

Fig. 6. (a) Output peak intensity of the mid-IR photodetector (PDAVJ10, Thorlabs) as a function of the input power of a $4.2 \mu \mathrm{m}$ femtosecond laser. (b) The impulse function of the photodetector measured in the linear region of (a), with the average over 1000 pulses. (c) Fourier spectrum of the impulse function in (b). (d) The Fourier spectrum of a stimulated Raman laser pulse based on the experimental parameters, where the pump energy is set to a maximum value of $\sim 80 \mu \mathrm{J}$, and $\mathrm{H}_{2}$ pressure to 15 bar. The inset of (d) is the corresponding pulse profile. (e) The measured pulse profile of a $4.22 \mu \mathrm{m}$ Raman laser (red curve), as well as the deconvoluted result (black curve). (f) Evolution of deconvoluted average pulse area and peak intensity as a function of the input power of the $4.22 \mu \mathrm{m}$ Raman laser (The output Raman laser from HCF is fixed at $132 \mathrm{~mW}$, and the power coupled to photodetector is precisely attenuated through a polarizer).

cascade laser which is known for its low-noise level and narrow linewidth.

In conclusion, we investigated the noise and long-term stability of an $\mathrm{H}_{2}$-filled Raman laser in both the near- and mid-IR spectral regions. Both REN and RIN of the presented IR Raman lasers continuously decrease with increasing pulse energy. At the same Raman laser power level, the RIN is always lower than the REN. The mid-IR Raman laser emission is associated with a large amount of heat release and long-term stability monitoring demonstrated some drift in the pulse peak intensity and pulse energy over time. Both the relatively high noise level and long-term drift could be underlying issues for the emerging IR gas-filled Raman lasers, despite their promising advantages of high quantum efficiency and both high energy and the high power laser emission.

\section{Appendix A}

In this experiment, the measured pulse profile of the $4.22 \mu \mathrm{m}$ Raman laser has a low accuracy because the $\sim 3$ ns rise time of the mid-IR photodetector is comparable with the pulse duration of the Raman laser. Since the photodetector with a much shorter rise time is not commercially available, here we use the deconvolution method to overcome this technical limitation and improve the accuracy of the pulse profile.

It is known that the output signal $O(t)$ from a photodetector is the convolution of the input signal $I(\mathrm{t})$ and the impulse function
$R(t)$ of the photodetector:

$$
O(t)=\int_{-\infty}^{\tau} I(\tau) R(t-\tau) d \tau
$$

We denote the Fourier transformation of $O(t), I(t)$, and $R(t)$ as $\tilde{\mathrm{O}}(\omega), \tilde{\mathrm{I}}(\omega)$, and $\tilde{\mathrm{R}}(\omega)$, respectively. From the convolution theorem, the Fourier transform of $O(t)$ can be expressed as:

$$
\tilde{\mathrm{O}}(\omega)=\tilde{\mathrm{I}}(\omega) \tilde{\mathrm{R}}(\omega)
$$

(1) indicates that $\tilde{I}(\omega)$ could be calculated by $\tilde{O}(\omega) / \tilde{R}(\omega)$, and then $I(t)$ could be retrieved from $\tilde{\mathrm{I}}(\omega)$ through the inverse Fourier transformation.

There are three prerequisites for the accurate retrieving of the $I(t):$

1) The impulse function $R(t)$ needs to be known. Here, we use a $\sim 250$ fs linearly polarized laser at $4.22 \mu \mathrm{m}$ to accurately measure the $R(t)$. This femtosecond laser is generated by an optical parametric amplifier (OPA) based on a $10 \mathrm{~mm}$ periodically-poled fan-out $\mathrm{MgO}: \mathrm{LiNbO}_{3}$ crystal. First, the power of the OPA laser is precisely controlled by a polarizer to search the linear region of the photodetector, as shown by Fig. 6 (a). With the increase in average power, it can be seen that the pulse peak intensity slowly increases until it reaches a saturation level. Then, the impulse function is measured by controlling the peak intensity to the center of the linear region, i.e., $\sim 14 \mathrm{mV}$. The measured profile of the impulse function is presented in Fig. 6(b), where 1000 pulses are averaged to smooth the background noise. Fig. 6(c) shows the Fourier transform spectrum of the impulse function in Fig. 6(b), where its maximum frequency $f_{\text {impulse_max }}$ is $\sim 200 \mathrm{MHz}$. 
2) According to the expression of $\tilde{O}(\omega)=\tilde{I}(\omega) \tilde{R}(\omega)$, in order to include the complete information of $\tilde{I}(\omega)$ into the output signal of $\tilde{O}(\omega)$, the frequency range of $\tilde{\mathrm{I}}(\omega)$ should be comparable or less than $\tilde{R}(\omega)$. This point is analyzed by comparing the Fourier spectrum of a typical simulated Raman laser pulse profile with the spectrum of $R(\mathrm{t})$. The simulation model is referred to [33], and the parameters are consistent with the experiment setup. The quantum noise model is referred to in [25]. The result is presented in Fig. 6(d), where one can see that the Raman laser pulse (simulated at 15 bar $\mathrm{H}_{2}$ pressure and maximum pump pulse energy of $\sim 80 \mu \mathrm{J})$ and the measured $R(\mathrm{t})$ has a comparable frequency range, thus, indicating that the pulse profile of the Raman laser could be approximately retrieved. Inset of Fig. 6(d) presents the corresponding pulse profile with a pulse duration of $\sim 4.8 \mathrm{~ns}$.

3) Because the output signal from the photodetector is sampled by an oscilloscope, according to the Nyquist sampling theorem, the maximum frequency $\mathrm{f}_{\text {output_max }}$ of the output signal must be less than half of the sampling frequency of the oscilloscope. From the above analysis, the value of $\mathrm{f}_{\text {output_max }}$ must less than $200 \mathrm{MHz}$, whereas the sampling frequency of the oscilloscope is up to $40 \mathrm{GHz}$. Therefore, this condition is well satisfied.

Since the vibrational Raman laser in our experiment approximately satisfies the above all prerequisites, its pulse profile could be basically retrieved. Fig. 6(e) shows a typical comparison of the Raman pulse profiles before and after deconvolution. Compared with the pulse profile before deconvolution with an obvious oscillation at its trailing edge region, the one after deconvolution has a reasonable Gauss-like pulse profile.

The correctness of this method is verified by investigating the evolution of the deconvoluted pulse area and peak intensity as a function of the average Raman laser power, as shown in Fig. 6(f). It should be noted here that the gas-filled Raman laser operates at a high quantum efficiency of $\sim 70 \%$ and pulse energy of $\sim 16 \mu \mathrm{J}$ at the $\mathrm{H}_{2}$ pressure of 15 bar, to maximally minimize the noise. The power coupling into the photodetector is precisely attenuated by a polarizer. The measured results show that both the peak intensity and pulse area initially increase in a linear dependence on the input power, and gradually deviates from the linear region because of the saturation effect of the photodetector. This indicates that the noise of both pulse energy and peak power of the Raman laser could be correctly and respectively reflected by the pulse area and peak intensity measured from the oscilloscope, as long as the Raman power is attenuated to the linear region.

\section{Appendix B}

The $4.22 \mu \mathrm{m}$ Raman laser in our experiment shows a high PER of over $-20 \mathrm{~dB}$ because of the use of a linear polarized pump as well as the weak birefringence of the ARHCF. As an example, the measured PER of the Raman laser was up to $-27 \mathrm{~dB}$ and $-22 \mathrm{~dB}$ at average powers of $132.8 \mathrm{~mW}$ and $32.3 \mathrm{~mW}$, respectively, as shown in Fig. 7(a) and 7(b). The PER of the vibrational Raman laser with lower power could
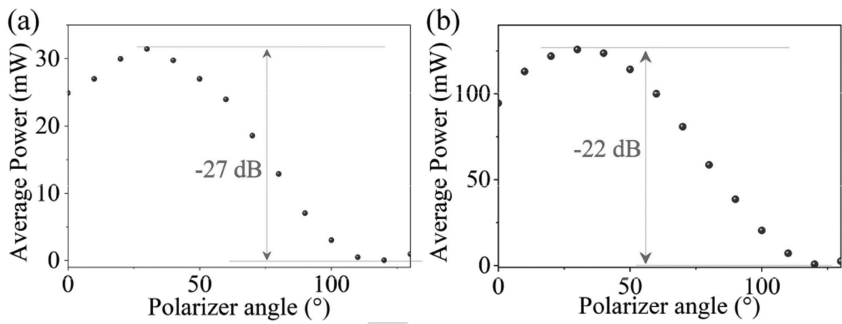

Fig. 7. PER measurement of the $4.22 \mu \mathrm{m}$ Raman laser at the average power of (a) $132.8 \mathrm{~mW}$ and (b) $32.3 \mathrm{~mW}$, respectively.
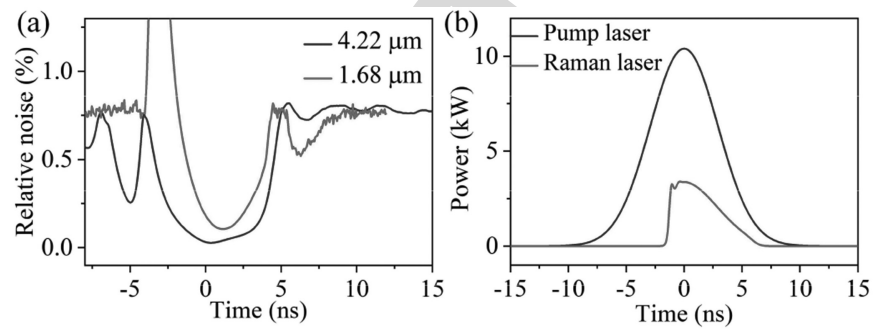

Fig. 8. (a) Instant noise distributions of the $4.22 \mu \mathrm{m}$ vibrational Raman laser and $1.68 \mu \mathrm{m}$ rotational Raman laser from the pulse leading edge to the trailing edge region. The zero time position is the position where the pulse peak power most probably appears. (b) A simulated Raman laser pulse and its corresponding pump pulse at the maximum pump energy of $\sim 80 \mu \mathrm{J}$ and $\mathrm{H}_{2}$ pressure of 15 bar.

not be accurately measured since the minimum power after the polarizer approaches the background noise level of the thermal power meter.

\section{Appendix C}

Fig. 8(a) shows the relative noise distributions of the instant pulse intensity from the pulse leading edge to the trailing edge region, for both the $1.68 \mu \mathrm{m}$ rotational Raman laser and $4.22 \mu \mathrm{m}$ vibrational Raman laser, at the same quantum efficiency of $\sim 45 \%$. 10000 pulses are used for each case. The statistics for the $4.22 \mu \mathrm{m}$ Raman laser were computed using the pulses after deconvolution (see Appendix A). The time at zero is the position where the pulse peak most probably appears. It can be seen that the pulse peak region has a lower noise level when compared to the pulse edge regions, indicating that the relative noise of the pulse peak intensity is lower than the pulse energy. This could be attributed to the dependence of the SRS efficiency on the laser intensity, as indicated by the simulation results in Fig. 8(b), where a typical Raman pulse and its corresponding pump pulse are presented for comparison. It can be seen that the peak regions of the Raman laser and pump laser are overlapped, because of the negligible dispersion effect of the gas-filled ARHCF. In this case, the peak region of the Raman pulse has higher SRS efficiency and therefore lower noise level than the pulse edge regions.

\section{Appendix D}

We observed that the coupling loss from the pump laser to the ARHCF can lead to power drift of the coupled pump power due to the heat release. In order to exclude the influence of the coupling efficiency drift during this experiment, the setup was always warming-up over 1 hour before the measurement, so that 

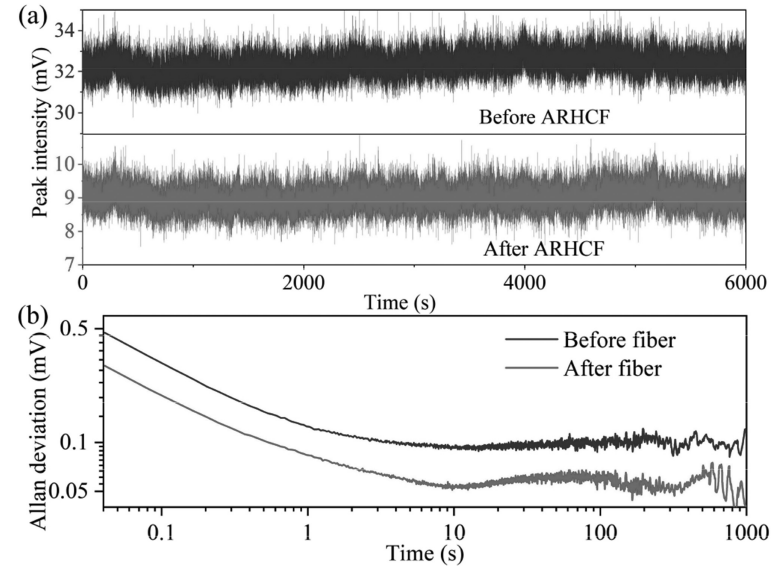

Fig. 9. (a) Long-term stability monitoring of the pump pulse peak intensities before and after the ARHCF by filling ambient air. Pump power was set to the maximum value of $1.4 \mathrm{~W}$. These results were recorded after one hours warmingup of the experiment system. (b) The calculated Allan deviations of the signals in (a).

the heat distribution can reach an equilibrium state. For better clarity, Fig. 9(a) shows a comparison of the long-term monitoring of pump peak intensities before and after the ARHCF. Two near-IR photodetectors (10Gbps PIN-TIA Detector, Nortel, Oclaro; and DET01CFC, Thorlabs) were placed before and after the ARHCF respectively, to simultaneously record the pulse peak intensities. This measurement was implemented by filling ambient air into the ARHCF, to avoid Raman laser-induced noise and drift. The pump laser was set to the maximum power of $1.4 \mathrm{~W}$. The recording started after one-hour warming-up. It can be seen that the signals before and after the ARHCF exhibit a similar fluctuation. This can also be reflected by the calculated Allan deviations in Fig. 9(b), where both curves show a similar evolution tendency as a function of time, and present a common inflection point at $\sim 10 \mathrm{~s}$, indicating that there is no coupling efficiency drift.

\section{ACKNOWLEDGMENT}

The authors would like to thank Callum R. Smith and Shreesha Rao Delanthabettu Shivarama for discussions of noise measurement. The authors also thank Christian R. Petersen for providing the mid-IR OPA.

\section{REFERENCES}

[1] F. Benabid, J. C. Knight, G. Antonopoulos, and P. S. J. Russell, "Stimulated raman scattering in hydrogen-filled hollow-core photonic crystal fiber," Science, vol. 298, no. 5592, pp. 399-402, Oct. 2002.

[2] F. Benabid, F. Couny, J. C. Knight, T. A. Birks, and P. S. J. Russell, "Compact, stable and efficient all-fibre gas cells using hollow-core photonic crystal fibres," Nature, vol. 434, no. 7032, pp. 488-491, Mar. 2005.

[3] C. Markos, J. C. Travers, A. Abdolvand, B. J. Eggleton, and O. Bang, "Hybrid photonic-crystal fiber," Rev. Mod. Phys., vol. 89, no. 4, Nov. 2017, Art. no. 45003.

[4] A. D. Pryamikov, A. S. Biriukov, A. F. Kosolapov, V. G. Plotnichenko, S. L. Semjonov, and E. M. Dianov, "Demonstration of a waveguide regime for a silica hollow - core microstructured optical fiber with a negative curvature of the core boundary in the spectral region $>3.5 \mu \mathrm{m}$," Opt. Exp., vol. 19, no. 2, pp. 1441-1448, Jan. 2011.
[5] M. S. Habib, J. E. Antonio-Lopez, C. Markos, A. Schülzgen, and R. Amezcua-Correa, "Single-mode, low loss hollow-core anti-resonant fiber designs," Opt. Exp., vol. 27, no. 4, Feb. 2019. Art. no. 3824.

[6] M. Klimczak et al., "Nested capillary anti-resonant silica fiber with midinfrared transmission and low bending sensitivity at $4000 \mathrm{~nm}$," Opt. Lett., vol. 44, no. 17, pp. 4395-4398, Aug. 2019.

[7] M. S. Astapovich, A. V. Gladyshev, M. M. Khudyakov, A. F. Kosolapov, M. E. Likhachev, and I. A. Bufetov, "Watt-level nanosecond 4.42- $\mu \mathrm{m}$ Raman laser based on silica fiber," IEEE Photonic. Technol. Lett., vol. 31, no. 1, pp. 78-81, Jan. 2019.

[8] L. Cao, S. Gao, Z. Peng, X. Wang, Y. Wang, and P. Wang, "High peak power $2.8 \mu \mathrm{m}$ Raman laser in a methane-filled negative-curvature fiber," Opt. Exp., vol. 26, no. 5, pp. 5609-5615, Feb. 2018.

[9] A. Gladyshev, Y. Yatsenko, A. Kolyadin, V. Kompanets, and I. Bufetov, "Mid-infrared 10- $\mu \mathrm{J}$-level sub-picosecond pulse generation via stimulated Raman scattering in a gas-filled revolver fiber," Opt. Mater. Exp., vol. 10, no. 12, pp. 3081-3089, Dec. 2020.

[10] A. V. Gladyshev et al., "4.4- $\mu$ m Raman laser based on hollow-core silica fibre," Quantum Electron, vol. 47, no. 5, pp. 491-494, Apr. 2017

[11] Y. Wang et al., "High pulse energy and quantum efficiency mid-infrared gas Raman fiber laser targeting $\mathrm{CO}_{2}$ absorption at $4.2 \mu \mathrm{m}$," Opt. Lett., vol. 45, no. 7, pp. 1938-1941, Mar. 2020.

[12] M. K. Mridha, D. Novoa, S. T. Bauerschmidt, A. Abdolvand, and P. St. J. Russell, "Generation of a vacuum ultraviolet to visible Raman frequency comb in $\mathrm{H}_{2}$-filled kagomé photonic crystal fiber," Opt. Lett., vol. 41, no. 12 , pp. 2811-2814, Jun. 2016.

[13] K. Krupa, K. Baudin, A. Parriaux, G. Fanjoux, and G. Millot, "Intense stimulated Raman scattering in $\mathrm{CO}_{2}$-filled hollow-core fibers," Opt. Lett., vol. 44, no. 21, pp. 5318-5321, Nov. 2019.

[14] Z. Li, W. Huang, Y. Cui, and Z. Wang, "Efficient mid-infrared cascade Raman source in methane-filled hollow-core fibers operating at $2.8 \mu \mathrm{m}$,' Opt. Lett., vol. 43, no. 19, pp. 4671-4674, Oct. 2018.

[15] Y. Chen, Z. Wang, B. Gu, F. Yu, and Q. Lu, "Achieving a $1.5 \mu \mathrm{m}$ fiber gas Raman laser source with about $400 \mathrm{~kW}$ of peak power and a $6.3 \mathrm{GHz}$ linewidth," Opt. Lett., vol. 41, no. 21, pp. 5118-5121, Nov. 2016.

[16] M. G. Raymer, I. A. Walmsley, J. Mostowski, and B. Sobolewska, "Quantum theory of spatial and temporal coherence properties of stimulated Raman scattering," Phys. Rev. A, vol. 32, no. 1, pp. 332-344, Jul. 1985.

[17] K. RząŻewski, M. Lewenstein, and M. G. Raymer, "Statistics of stimulated Stokes pulse energies in the steady-state regime," Opt. Commun., vol. 43 , no. 6, pp. 451-454, Nov. 1982.

[18] M. G. Raymer, K. Rzażewski, and J. Mostowski, "Pulse-energy statistics in stimulated Raman scattering," Opt. Lett., vol. 7, no. 2, pp. 71-73, Feb. 1982.

[19] M. G. Raymer and I. A. Walmsley, III The Quantum Coherence Properties of Stimulated Raman scattering. vol. 28, E. B. T.-P. O. Wolf, ed. Amsterdam, The Netherlands; New York: Elsevier, 1990, pp. 181-270.

[20] D. C. MacPherson, R. C. Swanson, and J. L. Carlsten, "Quantum fluctuations in the stimulated-Raman-scattering linewidth," Phys. Rev. Lett. vol. 61, no. 1, pp. 66-69, Jul. 1988.

[21] I. A. Walmsley and M. G. Raymer, "Observation of macroscopic quantum fluctuations in stimulated Raman scattering," Phys. Rev. Lett., vol. 50, no. 13 , pp. 962-965, Mar. 1983

[22] I. A. Walmsley and M. G. Raymer, "Experimental study of the macroscopic quantum fluctuations of partially coherent stimulated Raman scattering," Phys. Rev. A, vol. 33, no. 1, pp. 382-390, Jan. 1986

[23] R. Stolen, "Polarization effects in fiber Raman and Brillouin lasers," IEEE J. Quantum Electron., vol. 15, no. 10, pp. 1157-1160, Oct. 1979.

[24] I. A. Walmsley, M. G. Raymer, T. Sizer, I. N. Duling, and J. D. Kafka, "Stabilization of Stokes pulse energies in the nonlinear regime of stimulated Raman scattering," Opt. Commun., vol. 53, no. 2, pp. 137-140, Feb. 1985

[25] E. Landahl, D. Baiocchi, and J. R. Thompson, "A simple analytic model for noise shaping by an optical fiber Raman generator," Opt. Commun., vol. 150, no. 1, pp. 339-347, May. 1998.

[26] P. Werle, R. Mücke, and F. Slemr, "The limits of signal averaging in atmospheric trace-gas monitoring by tunable diode-laser absorption spectroscopy (TDLAS)," Appl. Phys. B., vol. 57, no. 2. pp. 131-139, Aug. 1993

[27] D. V. Land, A. P. Levick, and J. W. Hand, "The use of the Allan deviation for the measurement of the noise and drift performance of microwave radiometers," Meas. Sci. Technol., vol. 18, no. 7, pp. 1917-1928, May. 2007.

[28] P. Zhao et al., "Mode-phase-difference photothermal spectroscopy for gas detection with an anti-resonant hollow-core optical fiber," Nat. Commun., vol. 11, no. 1, pp. 1-8, Feb. 2020.

[29] Y. Wang et al., "Mid-infrared photoacoustic gas monitoring driven by a gas-filled hollow-core fiber laser," Sci. Rep., vol. 11, no. 1, pp. 1-8, Feb. 2021 
[30] T. Schmid, "Photoacoustic spectroscopy for process analysis," Anal Bioanal. Chem., vol. 384, no. 5, pp. 1071-1086, Jun. 2006.

[31] J. Hodgkinson, R. Smith, W. O. Ho, J. R. Saffell, and R. P. Tatam, "Nondispersive infra-red (NDIR) measurement of carbon dioxide at $4.2 \mu \mathrm{m}$ in a compact and optically efficient sensor," Sensors Actuators B Chem, vol. 186, pp. 580-588, Sep. 2013.

[32] N. Vermeulen, C. Debaes, P. Muys, and H. Thienpont, "Mitigating heat dissipation in Raman lasers using coherent anti-Stokes Raman scattering,' Phys. Rev. Lett., vol. 99, no. 9, Aug. 2007, Art. no. 93903

[33] G. Hilfer and C. R. Menyuk, "Stimulated Raman scattering in the transient limit," J. Opt. Soc. Am. B, vol. 7, no. 5, pp. 739-749, May. 1990.

Yazhou Wang received the B.Sc. degree in physics from Southwest University, the M.Sc. degree in 3D displaying from Sichuan University, and the Ph.D. degree in nonlinear fiber optics from the University of Electronic Science and Technology of China. During the Ph.D., he was a Visiting Student with the Technical University of Denmark in 2018, with the study on air plasma based $\mathrm{THz}$ generation. He received the Ph.D. degree in 2019, he joined Technical University of Denmark as a Post-Doc. His currently study focuses on infrared gas-filled fiber Raman laser design and applications in gas detection. He is a member of OSA and SPIE society.

Abubakar Isa Adamu (Member, IEEE) received the B.Sc. degree in engineering physics from Gaziantep University, Turkey and the M.Sc. degree in material science and nanotechnology from Bilkent University, Turkey, where he worked on development of artificial nose, for sensing of toxic gases with National Nanotechnology Research Centre (UNAM), Turkey. He was a Visiting Student with the University of Chicago, USA, in 2013, where he learnt therapeutic modalities in radiation oncology. He was a Guest Research Student with the University of Twente, The Netherlands, in 2015, where he worked on membranes for next-generation EUV lithography. Currently, he is a Manufacturing Engineer with Lumenisity Ltd, U.K.

He received the Doctorate degree in 2020 from the Technical University of Denmark in photonics engineering, where we worked on hollow-core optical fibres, ultrafast nonlinear optics, supercontinuum laser and fabrication of hybrid speciality fibres. Dr. Adamu is a member of OSA, EPS, and SPIE. He was recently awarded the Outstanding Student Paper Award at SPIE Photonics West, 2019, San Francisco, USA and Best Research Poster Award by Danish Optical Society (DOPS).

Manoj K. Dasa was born in Hyderabad, India, in 1991. He received the B.Tech degree in electronics and communications engineering from Jawaharlal Nehru Technological University, Hyderabad, India, and the M.Sc. degree in electrical communications engineering with a specialization in optoelectronics from the University of Kassel, Germany, in April 2016. In February 2017, he joined the Technical University of Denmark as a Marie Curie Ph.D. Fellow with the Department of Photonics Engineering. His Ph.D. degree focused on developing high-pulse energy supercontinuum sources for multispectral photoacoustic applications in the extended near-infrared region. His research interest includes supercontinuum lasers, biomedical imaging, and spectroscopy.

Jose E. Antonio-Lopez received the Ph.D. degree from the Instituto Nacional de Astrofísica, Óptica y Electrónica (INAOE), San Andres Cholula, Mexico, in 2012, with the work design and fabrication of photonic devices based on multimode interference. He is a Research Scientist with the College of Optics and Photonics (CREOL), University of Central Florida (UCF), Orlando, FL, USA. His research interests include design, fabrication, and use of special fibers
Md. Selim Habib (Senior Member, IEEE) received the B.Sc. and M.Sc. degrees in electrical and electronic engineering from the Rajshahi University of Engineering and Technology, Rajshahi, Bangladesh, in 2008 and 2012, respectively. He received the Ph.D. degree from Technical University of Denmark (DTU) in 2017. After finishing his Ph.D., he joined as a Postdoctoral Researcher in Fibers Sensors and Supercontinuum Group with the Department of Photonics Engineering, DTU. After finishing his Postdoctoral Fellowship with DTU, he worked as a Postdoctoral Research Associate with CREOL, The College of Optics and Photonics, University of Central Florida, USA, from 2017 to 2019. $\mathrm{He}$ is currently an Assistant Professor of Electrical and Computer Engineering with Florida Polytechnic University, USA. He has authored or coauthored more than 50 articles in referred journals. His research mainly focuses on design, fabrication, and characterization of low loss hollow-core fiber in the near-IR to mid-IR, light gas nonlinear interaction in hollow-core fibers, supercontinuum generation, and multi-mode nonlinear optics.

Dr. Habib is a Senior Member of Institute of Electrical and Electronics Engineers (IEEE), Optical Society of America (OSA) Early Careers Member, and Executive officer of OSA Fiber modeling and Fabrication group. Dr. Habib is an Associate Editor of IEEE ACCESS, and Feature Editor of Applied Optics (OSA). He was the recipient of the University Gold Medal Award from Rajshahi University of Engineering and Technology in 2014.

Rodrigo Amezcua Correa received the Ph.D. degree from the Optoelectronics Research Centre University of Southampton, Southampton, U.K. Since 2011, he has been with CREOL, The College of Optics and Photonics, University of Central Florida, Orlando, FL, USA. He is currently an Associate Professor of optics and photonics with CREOL. His research interests include optical fibers and fiber devices, fiber laser systems, fiber optic sensing, and optical communications.

Ole Bang received the M.Sc. degree in electrical engineering from the Technical University of Denmark (DTU), Kongens Lyngby, Denmark, in 1992 and the $\mathrm{Ph} . \mathrm{D}$. degree in nonlinear physics from DTU in 1993. From 1993 to 1995, he was a Postdoc with the Laboratoire de Physique, Ecole Normale Supérieure de Lyon, Lyon, France, working on discrete physical models of the nonlinear dynamics of biomolecules. From 1995 to 1999, he was a Research Fellow with Optical Sciences Centre, Australian National University, Canberra, ACT, Australia, where he worked theoretically on nonlinear optics, in particular, on solitons and modulational instability in materials with a quadratic nonlinearity and quasi-phase-matching gratings. From 1999 to 2003, he was an Associate Professor with the Department of Informatics and Mathematical Modeling, DTU. Since 2003, he has been with DTU Fotonik, Department of Photonics Engineering, DTU, where he became Professor in 2011, and is currently heading the Fiber Sensors \& Supercontinuum group. His current research interests include microstructured optical fibers in silica, polymer, and soft glasses, fiber optical sensors for sensing biomolecules, $\mathrm{pH}$, Oxygen, humidity, stress/strain, in particular using Fiber Bragg gratings, and the fabrication and application of supercontinuum sources, in particular in spectroscopy and imaging, such as OCT and optical-resolution photo-acoustic microscopy. He has been a consultant in supercontinuum sources with NKT Photonics since 2009. In 2015, he established the company SHUTE Sensing Solutions to market polymer fiber sensors and in 2018, he established the company NORBLIS to market mid-IR supercontinuum lasers and OCT systems.

Christos Markos received the B.Eng. (Hons) and M.Sc. (with Distinction) degree from the University of Liverpool in Electrical Engineering and Electronics, in 2007 and 2008. He continued his studies as Ph.D. student with the National Hellenic Research Foundation, Theoretical and Physical Chemistry Institute, Athens and received the Ph.D. degree in optics/optoelectronics in 2013. His main research activities are broad within the field of optical materials and nonlinear optics and particularly in gas-filled hollow-core fiber lasers, chalcogenide glasses, imaging and multimaterial fiber optics towards development of novel neural interfaces and optoelectronic smart systems. He has joined and worked with several distinguished research groups in USA and Europe including the Multi-material Optical Fiber Devices Group in the College of Optics and Photonics (CREOL), USA and Mid-Infrared Photonics Group with the University of Nottingham, U.K. He has established three state-of-the-art laboratories at DTU Fotonik for soft-glass synthesis, extrusion and fiber fabrication and since 2018 holds the position of Associate Professor. He is a member of OSA and SPIE society, Associate Editor of Optical Material Express journal and co-founder of NORBLIS. 